\title{
THE HAHN EMBEDDING THEOREM FOR ABELIAN LATTICE-ORDERED GROUPS
}

\author{
BY \\ PAUL CONRAD, JOHN HARVEY AND CHARLES HOLLAND(1)
}

1. Introduction. In 1939, Lorenzen [13] proved that an abelian lattice-ordered group (notation $l$-group) can be embedded into a large cardinal sum of linearly ordered groups (notation o-groups) as a sublattice and as a subdirect sum. Since then Birkhoff [2], Jaffard [10;11], Ribenboim [16;17], and others have reproved and refined this result. Sik $[18 ; 19]$ extends the Lorenzen embedding to a class of non-abelian l-groups. However, none of these refinements sheds any light on the structure of an $o$-group.

In 1907, Hahn [9] proved his embedding theorem for abelian o-groups, and Clifford [4] and Banaschewski [1] have given much simpler proofs of this result. Conrad [5] generalized the Hahn embedding so that it applies to abelian partially ordered groups (notation po-groups), and Gravett [7;8] has extended Conrad's results to po-sets. These generalizations of Hahn's embedding, when applied to an abelian $l$-group, do not usually embed it into an $l$-group.

The main result in this paper is a natural method for embedding an abelian $l$-group $A$ onto a sublattice of an $l$-group of real valued functions. This embedding reduces to the Hahn embedding in the special case when $A$ is an $o$-group, and the Lorenzen embedding with most of its refinements is an easy corollary of this embedding.

For the remainder of this section let $G$ be an abelian $l$-group, and let $\Gamma_{1}$ be the set of all pairs $\left(G^{\gamma}, G_{\gamma}\right)$ of $l$-ideals of $G$ such that $G_{\gamma}$ is a maximal $l$-ideal of $G$ with respect to not containing some element $g$ of $G$, and $G^{\gamma}$ is the unique $l$-ideal of $G$ that covers $G_{\gamma}$ (Theorem 4.1). We shall frequently identify the pair $\left(G^{\gamma}, G_{\gamma}\right)$ with $\gamma$. For each $\gamma$ in $\Gamma_{1}, G^{\gamma} / G_{\gamma}$ is an Archimedean $o$-group, and hence it is $o$-isomorphic to a subgroup of the additive group of real numbers with the natural order. Moreover, each $G / G_{\gamma}$ is an $o$-group (Theorem 4.1). For $\alpha$ and $\beta$ in $\Gamma_{1}$ we define $\alpha \geqq \beta$ if $\alpha=\beta$ or $G_{\alpha} \supseteq G^{\beta}$. It follows that $\Gamma_{1}$ is a $p o$-set with the following two properties:

$(\alpha)$ For each $g \neq 0$ in $G$ there exists at least one $\gamma$ in $\Gamma_{1}$ such that $g$ belongs to $G^{\gamma}$ but not to $G_{\gamma} ; \gamma$ is called a value of $g$.

( $\beta$ ) If $g \notin G^{\gamma}$, then there exists a $\beta>\gamma\left(\beta \in \Gamma_{1}\right)$ such that $g$ belongs to $G^{\beta}$, but not to $G_{\beta}$.

Received by the editors February 12, 1962.

(1) This work was supported by a grant from the National Science Foundation. 
A subset of $\Gamma_{1}$ that satisfies $(\alpha)$ and $(\beta)$ is said to be plenary.

Let $\Delta$ be a plenary subset of $\Gamma_{1}$, and let $L$ be the large direct sum of the $o$-groups $G^{\delta} / G_{\delta}(\delta \in \Delta)$. Let $V=V\left(\Delta, G^{\delta} / G_{\delta}\right)$ be the subgroup of $L$ consisting of all vectors $v=\left(\cdots, v_{\delta}, \cdots\right)$ such that $v_{\delta}=0$ for all $\delta$ in $\Delta$ with the exception of a set that satisfies the maximum condition. If $v_{\alpha} \neq 0$ and $v_{\beta}=0$ for all $\beta>\alpha$, then $v_{\alpha}$ is called a maximal component of $v$. We define that $v \in V$ is positive if all its maximal components are positive.

We prove that $V$ is an $l$-group (Theorem 2.2 and Lemma 4.3), and Theorem 4.2 states that if $G$ is divisible and $\Delta$ is a plenary subset of $\Gamma_{1}$, then there exists an isomorphism $\phi$ of $G$ into $V$ such that $\delta \in \Delta$ is a value of $g$ in $G$ if and only if $(g \phi)_{\delta}$ is a maximal component of $g \phi$, and in this case $(g \phi)_{\delta}=g+G_{\delta}$. Such an isomorphism $\phi$ is called a $v$-isomorphism, and every $v$-isomorphism of $G$ into $V$ is an $l$-isomorphism. In particular, $G \phi$ is a sublattice of $V$. Also, plenary subsets are exactly those subsets of $\Gamma_{1}$ that support $v$-isomorphisms (Theorem 3.1).

There is a natural $l$-isomorphism $\psi$ of $V$ onto a subdirect sum of a large cardinal sum of $o$-groups. Thus both the Lorenzen and the Hahn embeddings are corollaries of our embedding. If $\Gamma_{1}$ has a minimal plenary subset $\Delta$, then $\Delta$ is unique (Theorem 5.2), and if $G$ has a basis [6, p. 218], then there exists a unique minimal plenary subset $\Delta$ of $\Gamma_{1}$ (Theorem 5.5). If $G$ is divisible and has a basis, then $\phi \psi$ gives an irreducible representation of $G$ as a sublattice and a subdirect sum of a cardinal sum of $o$-groups where $\psi$ is a natural splitting of $\Delta$ into chains. Thus we recover a result of Jaffard [10, Theorem 4, p. 251]. If $G$ is Archimedean and $\Delta$ is a minimal plenary subset of $\Gamma_{1}$, then $G$ has a basis (Theorem 5.4).

In $\$ 5$ we prove that $G$ has a finite basis if and only if $\Gamma_{1}$ contains only a finite number of maximal chains. Thus we can recover the structure theorem for divisible abelian $l$-groups with a finite basis [6, Theorem 2.2 and Proposition 11.3] or [11]. We also derive (Theorem 5.4) a necessary and sufficient condition on $G$ for $\Gamma_{1}$ to admit a minimal plenary subset.

If $\Delta$ is a plenary subset of $\Gamma_{1}$ such that each nonzero element of $G$ has at most a finite number of values in $\Delta$, then the lattice of all $l$-ideals of $G$ is completely determined by $\Delta$ (Theorem 5.10 ).

We also show (Theorem 6.4) that an abelian $l$-group can be embedded as a subdirect sum and a sublattice of a large cardinal sum of subgroups of the real numbers if and only if the intersection of the maximal $l$-ideals is $\{0\}$. An example is given (due to Kaplansky) of an Archimedean l-group that cannot be so embedded.

Notation. The null set will always be denoted by $\square$. If $a$ and $b$ are elements of a $p o$-set, then $a \| b$ will denote that $a \geq b$ and $a \$ b$. If $S$ is a subset of a group $H$, then $[S]$ will denote the subgroup of $H$ that is generated by $S$. Lattice operations will be denoted by $\wedge$ and $\vee$, and set theoretic operations by $\cap$ and $\cup$. If $S$ and $T$ are subsets of a set, then $S \backslash T$ will denote the set of all elements in $S$ that are not in $T$. 
2. A Hahn-type po-group. Let $\Gamma$ be a po-set and for each $\gamma \in \Gamma$, let $H_{\gamma}$ be a nontrivial po-group. Let $V=V\left(\Gamma, H_{\gamma}\right)$ be the following subset of the large direct sum of the $H_{\gamma}$. An element $v=\left(\cdots, v_{\gamma}, \cdots\right)$ belongs to $V$ if and only if

$$
S_{v}=\left\{\gamma \in \Gamma \mid v_{\gamma} \neq 0\right\}
$$

contains no infinite ascending sequences. This is clearly equivalent to the maximum condition which is that every nonempty subset of $S_{v}$ contains at least one maximal element.

It is easy to verify that if $S$ and $T$ are subsets of $\Gamma$, each of which satisfy the maximum condition, then $S \cup T$ satisfies the maximum condition. Thus, it follows that $V$ is a subgroup of the large direct sum of the $H_{\gamma}$. For each $v \in V$, let

$$
\Gamma^{v}=\left\{\gamma \in \Gamma \mid v_{\gamma} \neq 0 \text { and } v_{\alpha}=0 \text { for all } \alpha>\gamma\right\} .
$$

The $v_{y}$ with $\gamma \in \Gamma^{v}$ are called maximal components of $v$. We define a nonzero element $v$ of $V$ to be positive if each maximal component $v_{y}$ of $v$ is positive with respect to the partial order on the group $H_{\gamma}$. We shall denote the identity of $V$ by $\theta$.

THEOREM 2.1. If $\Gamma$ is a po-set, if for each $\gamma \in \Gamma H_{\gamma}$ is a po-group, and if $V$ is partially ordered as above, then $V$ is a po-group.

Proof. Let $P$ be the set of positive elements of $V$. By definition $\theta \notin P$; hence, all we must show is that $P$ is a normal subsemigroup of $V$. If $u$ and $v$ are elements of $P$, then clearly $u+v \neq \theta$. Let $u_{\gamma}+v_{\gamma}$ be a maximal component of $u+v$. Without loss of generality we may assume that $u_{\gamma} \neq 0$. If $\gamma \notin \Gamma^{u}$, then there must exist $\alpha>\gamma$ such that $u_{\alpha}>0$ and $u_{\beta}=0$ for all $\beta>\alpha$. Since $u_{\alpha}+v_{\alpha}=0, v_{\alpha}<0$, and hence $\alpha \notin \Gamma^{v}$. Thus there must exist $\beta>\alpha$ such that $0<v_{\beta}$. But then $u_{\beta}+v_{\beta}=v_{\beta} \neq 0$, and this contradicts the assumption that $\gamma \notin \Gamma^{u+v}$. It follows that $\gamma \in \Gamma^{u}$, and so $0<u_{\gamma}$. Similarly, if $v_{\gamma} \neq 0$, then $0<v_{\gamma}$. Therefore $0<u_{\gamma}+v_{\gamma}$, and hence $u+v \in P$.

To prove that $P$ is normal, suppose that $u \in P$ and $v \in V$. If $\gamma \in \Gamma^{-v+u+v}$, then clearly $u_{\gamma} \neq 0$, and $u_{\beta}=0$ for all $\beta>\gamma$. Thus $\gamma \in \Gamma^{u}$, and hence, $u_{\gamma}>0$. Therefore $0<-v_{\gamma}+u_{\gamma}+v_{\gamma}$, and it follows that $-v+u+v \in P$.

THEOREM 2.2. The po-group $V$ is an l-group if and only if

(a) $H_{\gamma}$ is an o-group if $\gamma$ is not minimal in $\Gamma$, and otherwise, $H_{\gamma}$ is an l-group; and

(b) no pair of incomparable elements of $\Gamma$ have a common lower bound.

Proof. Throughout this argument for $a \in H_{y}$ let $\bar{a}$ denote the element of $V$ whose $\gamma$ th component is $a$ and all of whose other components are zero. We first suppose that $V$ is an $l$-group.

Assume (by way of contradiction) that for some $\gamma \in \Gamma, H_{\gamma}$ is not an $l$-group. Then [3, p. 215] there exists an element $a \in H_{\gamma}$ such that $a$ and 0 have no least 
upper bound in $H_{\gamma}$. If $\bar{a} \vee \theta=\theta$, then $\bar{a} \leqq \theta$, and thus, $a \leqq 0$, a contradiction. Therefore $\theta<\bar{a} \vee \theta$.

Suppose there exists a maximal component $b_{\alpha}$ of $\tilde{a} \vee \theta$ such that $\gamma<\alpha$. Then $0<b_{\alpha}$, and hence $\theta<\bar{a}+(\bar{a} \vee \theta)$. Also, since $\theta<\bar{a} \vee \theta, \bar{a}=\bar{a}+\theta<\bar{a}+(\bar{a} \vee \theta)$. Hence, $\vec{a} \vee \theta \leqq \bar{a}+(\bar{a} \vee \theta)$, and thus, $\theta \leqq \bar{a}$, a contradiction. Hence, there can exist no maximal component $b_{\alpha}$ of $\bar{a} \vee \theta$ such that $\gamma<\alpha$. Thus, since $\theta<\bar{a} \vee \theta$ and $\bar{a} \leqq \bar{a} \vee \theta$, it follows that $(\bar{a} \vee \theta)_{\gamma}$ is a maximal component of $\bar{a} \vee \theta$, that 0 $<(\bar{a} \vee \theta)_{\gamma}$, and that $a<(\bar{a} \vee \theta)_{\gamma}$. Now, since $a \vee 0$ does not exist, there exists $q \in G_{\gamma}$ such that $0<q<(\tilde{a} \vee \theta)_{\gamma}$ and $a<q$. Therefore, if we replace $(\tilde{a} \vee \theta)_{\gamma}$ by $q$ in $\bar{a} \vee \theta$, we get an element $v$ of $V$ such that $a<v, \theta<v$, and $v<\bar{a} \vee \theta$. a contradiction. Thus each $H_{\gamma}$ is an $l$-group.

If $\alpha$ and $\gamma$ are elements of $\Gamma$ and $\alpha<\gamma$, then $H_{\gamma}$ is an $o$-group. Otherwise, there exists $a \in H_{\gamma}$ such that $a \| 0$. As above, $(\bar{a} \vee \theta)_{\gamma}$ is a maximal component of $\bar{a} \vee \theta$, and clearly $a<a \vee 0=(\bar{a} \vee \theta)_{\gamma}$. Choose a negative element $c$ in $H_{\alpha}$. Then clearly $\bar{a}<\bar{c}+(\bar{a} \bigvee \theta)$ and $\theta<\bar{c}+(\bar{a} \bigvee \theta)$ because the lattice order on $V$ only depends upon the maximal components. But $\bar{c}<\theta$ implies $\bar{c}+(\bar{a} \bigvee \theta)<\bar{a} \bigvee \theta$, and this is a contradiction. Therefore, $H_{\gamma}$ is an $o$-group; hence (a) is satisfied whenever $V$ is an $l$-group.

Next assume that $\alpha, \beta$, and $\gamma$ are elements of $\Gamma$ such that $\gamma<\alpha, \gamma<\beta$ and $\alpha \| \beta$. Choose $0<a \in H_{\alpha}$ and $0<b \in H_{\beta}$, and let $v$ be any element of $V$ such that $\theta<v<\bar{a}$ and $v<\bar{b}$. For example, if $0<c \in H_{\gamma}$, then $\bar{c}$ is such an element. Let $0<v_{\delta}$ be a maximal component of $v$. If $\delta \$ \alpha$, then, since $0<\bar{a}-v, v_{\delta}<0$, a contradiction. Thus $\delta \leqq \alpha$, and $\delta \leqq \beta$, and since $\alpha \| \beta, \delta<\alpha$ and $\delta<\beta$, and no further restrictions are placed on $v$. It follows that $n v<\tilde{a}$ and $n v<\bar{b}$ for all positive integers $n$. Therefore $\bar{a} \wedge \bar{b}$ cannot exist, which contradicts the assumption that $V$ is an $l$-group. Thus (b) is satisfied whenever $V$ is an $l$-group.

Conversely, suppose that (a) and (b) are satisfied. It suffices to show that $v$ and $\theta$ have a least upper bound for each $v$ in $V$. Consider $\theta \neq v \in V$, and let $v_{\gamma}$ be a maximal component of $v$. If $\gamma$ is minimal in $\Gamma$, replace $v_{\gamma}$ by $v_{\gamma} \vee 0$, and if $\gamma$ is not minimal and $v_{\gamma}<0$, replace $v_{\beta}$ by 0 for all $\beta \leqq \gamma$. It follows by a straightforward argument that the resulting element is $v \vee \theta$. We next give another and somewhat more transparent way of describing $v \vee \theta$.

Let $M$ be a maximal chain of $\Gamma$, and let $V_{M}$ be the projection of $V$ on $M$, i.e.,

$$
V_{M}=\left\{v \in V \mid v_{\gamma}=0 \text { for all } \gamma \in \Gamma \backslash M\right\} .
$$

Condition (a) assures us that $V_{M}$ is an $l$-group. For, if $M$ contains no least element then $V_{M}$ is an $o$-group, and if $\gamma$ is the least element in $M$, then $V_{M}$ is a direct lexicographical extension [6, p. 214] of the l-group $H_{y}$ by an $o$-group. Hence, in any case, $V_{M}$ is an l-group.

Once again consider $\theta \neq v \in \Gamma$. For each maximal chain $M$ of $\Gamma$, let $v_{M}$ be the projection of $v$ upon $V_{M}$. It follows from condition (b) that $\theta<v$ if and only 
if $\theta \leqq v_{M}$ for all such $M$. For each maximal chain $M$ of $\Gamma$ and for each $\gamma \in M$ replace $v_{\gamma}$ by $\left(v_{M} \vee \theta\right)_{\gamma}$, and let $v^{*}$ be the resulting vector. Condition (b) assures us that the process is well defined, and it follows easily that $v^{*}=v \vee \theta$. This completes the proof of the theorem.

If $\Gamma$ is a po-set which satisfies condition (b) of Theorem 2.2, then we shall say that $\Gamma$ is a root system.

The following propositions are easily verified. Most of them are immediate consequences of Theorem 2.2.

(1) $V$ is an $o$-group if and only if $\Gamma$ is (totally) ordered and each $H_{\gamma}$ is an $o$-group.

(2) If $\Gamma$ is ordered and contains no least element, then $V$ is an l-group if and only if $V$ is an $o$-group.

(3) If $\Gamma$ is ordered and has a least element $\alpha$ and if $V$ is an $l$-group, then $V$ is an $o$-group if and only if $H_{\alpha}$ is an $o$-group.

(4) If $\Gamma$ has the trivial partial order, then

(i) $V$ is the large cardinal sum of the $H_{\gamma}$;

(ii) $V$ is an $l$-group if and only if each $H_{\gamma}$ is an $l$-group.

(5) If for each $\gamma \in \Gamma, H_{\gamma} \neq 0$ is an $o$-subgroup of the real numbers (under addition ordered in the usual way), then

(i) $V$ is an $o$-group if and only if $\Gamma$ is ordered. Such an $o$-group will be called a Hahn group;

(ii) $V$ is Archimedean [3, p. 255], if and only if $\Gamma$ is trivially ordered;

(iii) the projection of $V$ upon any chain in $\Gamma$ is a Hahn group.

3. Embedding theorem for $p o$-groups. Throughout this section, let $G$ be an abelian po-group which has the property that if $g \in G$ and $0 \leqq n g$ for some positive integer $n$, then $0 \leqq g$. Then $G$ is an abelian semi-closed po-group. Clearly, $G$ is a torsion-free group. Also, it is true that any lattice-ordered group is a po-group with this property.

Let $P=\{g \in G \mid 0<g\}$, and let $\bar{G}$ be the unique (abelian) divisible closure of $G$. Then $\bar{P}=\{\bar{g} \in \bar{G} \mid 0<n \bar{g} \in G$ for some integer $n>0\}$ defines a partial order on $\bar{G}$ which is an extension of the partial order defined on $G$, i.e., $P=\bar{P} \cap G$. Further, if $G$ is an $l$-group, then $G$ is an $l$-group and $G$ is a sublattice of $G$.

A subgroup $C$ of $G$ is said to be convex if

(i) $C$ is pure, and

(ii) $a<b<c$ implies that $b \in C$ whenever both $a$ and $c$ are elements of $C$.

In particular, if $G$ is divisible, then so are its convex subgroups and if $G$ is an $l$-group, then each $l$-ideal satisfies (i) and (ii).

Let $\Gamma$ be the set of all pairs of convex subgroups $\left(G^{\gamma}, G_{\gamma}\right)$ of $G$ such that $G^{\gamma}$ covers $G_{\gamma}$. If $G$ is nontrivial, then $\Gamma$ is nonempty [5, p. 22]. We shall frequently identify the pair $\left(G^{\gamma}, G_{\gamma}\right)$ with $\gamma$. For each $\gamma \in \Gamma, G^{\gamma} / G_{\gamma}$ is $o$-isomorphic to a subgroup of the real numbers (ordered as usual) unless it is trivially ordered, and in this 
case it is isomorphic to a subgroup of the additive group of rational numbers [5, p. 23]. For $\alpha$ and $\beta$ in $\Gamma$ define that $\alpha \leqq \beta$ if $G^{\alpha}=G^{\beta}$ and $G_{\alpha}=G_{\beta}$ or if $G^{\alpha} \subseteq G_{\beta}$. Then $\Gamma$ is a po-set. If $g \in G^{\delta} \backslash G_{\delta}$, then $\delta$ is said to be a value of $g$. Let $\Delta$ be a subset of $\Gamma$. The following two concepts are basic for our theory.

$\Delta$ is said to be plenary if it has the following two properties:

$(\alpha)$ Each nonzero element in $G$ has at least one value in $\Delta$.

( $\beta$ ) If $g \in G \backslash G^{\delta}$ for some $\delta \in \Delta$, then there exists a value of $g$ in $\Delta$ that exceeds $\delta$. In particular, $\Gamma$ itself is plenary.

An isomorphism $\phi$ of $G$ into $V\left(\Delta, G^{\delta} / G_{\delta}\right)$ is said to be valuation preserving (notation $v$-isomorphism) provided that it satisfies

(v) $\delta \in \Delta$ is a value of $g \in G$ if and only if $(g \phi)_{\delta}$ is a maximal component of $g \phi$, and in this case $(g \phi)_{\delta}=G_{\delta}+g$.

Suppose that $\phi$ is a $v$-isomorphism of $G$ into $V\left(\Delta, G^{\delta} / G_{\delta}\right)$ and consider $0<g \in G$. Clearly $G_{\delta}+g$ is positive for every value $\delta$ of $g$ in $\Delta$ and hence $g \phi$ is positive in $V$. If $\Delta=\Gamma$, then $\phi^{-1}$ also preserves order [5, p. 23].

THEOREM 3.1 (MAIN EMBEDDING THEOREM). Let $G$ be a divisible abelian pogroup and let $V=V\left(\Delta, G^{\delta} / G_{\delta}\right)$, where $\Delta$ is a subset of $\Gamma$. Then the following are equivalent:

(1) There exists a v-isomorphism of $G$ into $V$.

(2) There exists an isomorphism $\phi$ of $G$ into $V$ such that $\delta \in \Delta$ is a value of $g \in G$ if and only if $(g \phi)_{\delta}$ is a maximal component of $g \phi$.

(3) $\Delta$ is a plenary subset of $\Gamma$.

Proof. Clearly (1) implies (2). Suppose that (2) is satisfied and consider $0 \neq g \in G$. There exists a maximal component $(g \phi)_{\delta}$ of $g \phi$ in $V$, and hence by our assumption on $\phi, \delta \in \Delta$ is a value of $g$. Suppose that $g \in G \mid G^{\beta}$, where $\beta \in \Delta$, and consider $y \in G^{\beta} \backslash G_{\beta}$. Then $(y \phi)_{\beta} \neq 0$ and $((g+y) \phi)_{\beta}=(g \phi)_{\beta}+(y \phi)_{\beta}$, so we may assume without loss of generality that $((g+y) \phi)_{\beta} \neq 0$ (otherwise choose $-y$ instead of $y$ ). It follows that $(g+y) \phi$ has a maximal component $((g+y) \phi)_{\gamma}$ where $\gamma \geqq \beta$, and hence $g+y \in G^{\gamma} \mid G_{\gamma}$ where $\gamma \in \Delta$. Since $g \notin G^{\beta}$ and $y \in G^{\beta}$ it follows that $\gamma>\beta$. Thus $y \in G^{\beta} \subseteq G_{\gamma}$, and hence $g \in G^{\gamma} \mid G_{\gamma}$. Therefore $\Delta$ is a plenary subset of $\Gamma$ and hence (2) implies (3). Note that we have not made use of the hypothesis that $G$ is divisible.

The fact that (3) implies (1) was proved in [5] and [8]. Here we give an entirely different proof that makes use of the fact that $G$ is a rational vector space, and the following result of Banaschewski [1, p. 431]:

There exists a mapping $\pi$ of the set of all subspaces of $G$ into itself such that for all subspaces $A$ and $B$ of $G$

(i) $G=A \oplus \pi(A)$, and

(ii) if $A \subseteq B$, then $\pi(A) \supseteq \pi(B)$.

For $x \in G$ we define $x \phi \in V$ as follows: for each $\delta \in \Delta$, let 


$$
(x \phi)_{\delta}=x_{\delta}+G_{\delta},
$$

where $x=x_{\delta}+c_{\delta}, x_{\delta} \in G^{\delta}$ and $c_{\delta} \in \pi\left(G^{\delta}\right)$. hen clearly $\phi$ is a homomorphism of $G$ into the large direct sum of the $G^{\delta} / G_{\delta}$. For any nonzero element $x$ of $G$, let

$$
R_{x}=\left\{\delta \in \Delta \mid(x \phi)_{\delta} \neq G_{\delta}\right\} .
$$

If $\delta$ is a value of $x$, then $(x \phi)_{\delta}=x+G_{\delta} \neq G_{\delta}$. It follows that $R_{x}$ is nonempty, and hence $\phi$ is an isomorphism. To prove that $G \phi \subseteq V$, it suffices to show that every ascending sequence of $R_{x}$ has a greatest element.

Let $M$ be an ascending sequence $\delta_{1}<\delta_{2}<\cdots$ in $R_{x}$, and let $Q=\bigcup\left\{G^{\delta_{i}} \mid \delta_{i} \in M\right\}$. Then $G=Q \oplus \pi(Q)$ and $x=z+y$ where $z \in Q$ and $y \in \pi(Q)$; hence $z$ belongs to one of the $G^{\delta_{i}}$, say $G^{\delta_{t}}$. Suppose (by way of contradiction) that $\delta_{t}<\delta_{s} \in M$. Then $(z \phi)_{\delta_{s}}=G_{\delta_{s}}$ because $z \in G^{\delta_{t}} \subseteq G_{\delta_{s}}$, and $(y \phi)_{\delta_{s}}=G_{\delta_{s}}$ because $G^{\delta_{s}} \subseteq Q$ and hence $y \in \pi(Q) \subseteq \pi\left(G^{\delta_{s}}\right)$. Thus $(x \phi)_{\delta_{s}}=(z \phi)_{\delta_{s}}+(y \phi)_{\delta_{s}}=G_{\delta_{s}}$, but this means that $\delta_{s} \notin R_{x}$, a contradiction. Therefore, $\delta_{t}$ is the largest element of $M$.

If $x \in G^{\delta} \mid G_{\delta}$, then $(x \phi)_{\delta}=x+G_{\delta} \neq G_{\delta}$, and if $\delta<\alpha \in \Delta$, then $(x \phi)_{\alpha}=G_{\alpha}$ because $x \in G_{\alpha}$. Thus $(x \phi)_{\delta}$ is a maximal component of $x \phi$. Conversely suppose that $(x \phi)_{\delta}$ is a maximal component of $x \phi$. If $x \notin G^{\delta}$, then by property $(\beta)$ there exists a value $\alpha$ of $x$ such that $\delta<\alpha$, but then $(x \phi)_{\alpha}=x+G_{\alpha} \neq G_{\alpha}$, a contradiction. If $x \in G_{\delta}$, then $(x \phi)_{\delta}=G_{\delta}$, a contradiction. Therefore, $\delta$ is a value for $x$.

If $g$ is positive in $G$, then $g+G_{\delta}$ is positive in $G^{\delta} / G_{\delta}$ for all values $\delta$ of $g$, and hence all maximal components of $g \phi$ are positive. Thus $g \phi$ is positive, and this completes the proof of the theorem.

Note that if we only require $\Delta$ to have property $(\alpha)$, then $\phi$ is still an isomorphism, and if $\delta$ is a value of $g$, then $(g \phi)_{\delta}=g+G_{\delta}$ is a maximal component of $g \phi$. If $\Delta=\Gamma$, then, as noted above, $\phi^{-1}$ also preserves order. In particular, if $\Gamma$ is ordered, then we have Hahn's embedding theorem for divisible, abelian $o$-groups [9]. Also, the hypothesis that $G$ is divisible can be weakened in various ways. For example, it suffices to assume that all of the $G^{\delta} / G_{\delta}$ are divisible [5, p. 13].

Suppose that $\Delta \subseteq \Gamma$. Let $Q=\left\{G_{\delta} \mid\left(G^{\delta}, G_{\delta}\right) \in \Delta\right\}$. A subset $A$ of $G \times Q$ is an overlap if $\left(x_{1}, G_{\delta_{1}}\right) \in A,\left(x_{2}, G_{\delta_{2}}\right) \in A, G_{\delta_{1}} \subseteq G_{\delta} \in Q$, and $G_{\delta_{2}} \subseteq G_{\delta}$ imply that $x_{1}-x_{2} \in G_{\delta}$. An element $x_{0}$ of $G$ is a limit for the overlap $A$ if $\left(x, G_{\delta}\right) \in A$ implies that $x-x_{0} \in G_{\delta}$. The group $G$ is said to be overlap complete with respect to $\Delta$ if each overlap has a limit in $G$.

Suppose now that $G$ is divisible and that $\Delta$ is a plenary subset of $\Gamma$ and that $\phi$ is the isomorphism derived in Theorem 3.1. Then we have the following theorems:

THEOREM 3.2 (GRAVETt). The mapping $\phi$ is an isomorphism of $G$ onto $V\left(\Delta, G^{\delta} / G_{\delta}\right)$ if and only if $G$ is overlap complete with respect to $\Delta[8, p .14]$.

THEOREM 3.3. If $\phi$ and $\phi^{\prime}$ are v-isomorphisms of $G$ into $V\left(\Delta, G^{\delta} / G_{\delta}\right)$, then there exists an o-automorphism $\psi$ of $V$ such that $(G \phi) \psi=G \phi^{\prime}[5, p .28]$.

In general, $\Gamma$ contains proper plenary subsets. For example, for each $0 \neq g \in G$, 
let $\mathscr{C}_{g}$ be the collection of all convex subgroups of $G$ that are maximal with respect to not containing $g$. Then for each $G_{g} \in \mathscr{C}_{g}$ there exists a unique convex subgroup $G^{g}$ of $G$ that covers $G_{g}$, namely the intersection of all convex subgroups of $G$ that contain $G_{g}$ and $g$. Let $\Gamma_{1}$ be the set of all these pairs $\left(G^{g}, G_{g}\right), g \in G$. Then clearly $\Gamma_{1}$ is a plenary subset of $\Gamma$, and for $\alpha, \beta \in \Gamma_{1}$

and

$$
\alpha=\beta \text { if and only if } G_{\alpha}=G_{\beta}
$$

$$
\alpha<\beta \text { if and only if } G_{\alpha} \subset G_{\beta} .
$$

In the next section we show that if $G$ is an $l$-group, then $V\left(\Gamma_{1}, G^{g} / G_{g}\right)$ is also an $l$-group and every $v$-isomorphism of $G$ into $V$ is an $l$-isomorphism. The next theorem shows that $\Gamma_{1}$ is usually a proper subset of $\Gamma$.

THEOREM 3.4. If $G$ is a divisible abelian po-group, then $\Gamma_{1}=\Gamma$ if and only if $G=R_{x} \oplus A$, where $R_{x}$ is the group of all rational multiples of a fixed element $x \in G, A$ is an o-group and $g=r x+a \in G$ is positive if and only if $a$ is positive in $A$. In particular, if $G$ is an l-group, then $\Gamma=\Gamma_{1}$ if and only if $G$ is an o-group.

Since we make no use of the above results we shall omit the proofs.

4. Embedding theorem for $l$-groups. Throughout this section, let $G$ be an abelian $l$-group, and let $\Gamma$ be the set of all pairs $\left(G^{\gamma}, G_{\gamma}\right)$ of $l$-ideals of $G$ such that $G^{\gamma}$ covers $G_{\gamma}$. In general, $\Gamma$ fails to satisfy condition (b) of Theorem 2.2, and hence $V\left(\Gamma, G^{\gamma} / G_{\gamma}\right)$ is not an l-group. In this section, we obtain an embedding of $G$ as a sublattice of an l-group $V\left(\Delta, G^{\delta} / G_{\delta}\right)$, where $\Delta$ is a plenary subset of $\Gamma$, under the assumption that $G$ is divisible. By Theorem 2.2 we must find a plenary subset $\Delta$ of $\Gamma$ such that no pair of incomparable elements of $\Delta$ have a common lower bound. It is not difficult to show that

$$
\Lambda=\{\lambda \in \Gamma \mid \text { the elements of } \Gamma \text { that exceed } \lambda \text { form a chain }\}
$$

is a plenary subset of $\Gamma$ and that $V\left(\Lambda, G^{\lambda} / G_{\lambda}\right)$ is an l-group. However if $G$ is the cardinal sum of the rational numbers by the rational numbers, then $\Lambda=\Gamma \neq \Gamma_{1}$, and it follows from Theorem 4.3 that no $v$-isomorphism of $G$ into $V\left(\Lambda, G^{\lambda} / G_{\lambda}\right)$ is an $l$-isomorphism. Thus, in general, we must choose a proper subset of $\Lambda$.

If $G$ is a subdirectly irreducible abelian $l$-group, then $G$ is an $o$-group [3,p. 224]. Thus we have

LEMmA 4.1. If $G$ is an abelian l-group and if there is a nonzero l-ideal $A$ of $G$ such that $A \subseteq B$ for all nonzero l-ideals $B$ of $G$, then $G$ is an o-group.

THEOREM 4.1. Suppose that $G$ is an abelian l-group, and let $g$ be any nonzero element of $G$. If $C$ is any l-ideal of $G$ which is maximal with respect to not containing the element $g$, then $G / C$ is an o-group with a convex subgroup that 
covers zero. Thus there exists a unique l-ideal $C^{\prime}$ of $G$ that covers $C$, and $C^{\prime}$ is contained in every other l-ideal of $G$ that properly contains $C$.

Proof. Let $C^{\prime}$ be the intersection of all $l$-ideals of $G$ which contain $C$ and $g$. If $C^{\prime \prime}$ is an $l$-ideal of $G$ which properly contains $C$, then $g \in C^{\prime \prime}$, and hence $C^{\prime} \subseteq C^{\prime \prime}$. Thus $C^{\prime}$ covers $C$, and each proper $l$-ideal of $G / C$ must contain the $l$-ideal $C^{\prime} / C$. Therefore by Lemma $4.1, G / C$ is an $o$-group.

We shall call an $l$-ideal of $G$ regular if it is maximal with respect to not containing some element of $G$. Let

$$
\Gamma_{1}=\left\{\gamma \in \Gamma \mid G_{\gamma} \text { is regular }\right\} .
$$

Note that if $\gamma \in \Gamma_{1}$, then $G^{\gamma}$ is the unique $l$-ideal of $G$ that covers $G_{\gamma}$ (Theorem 4.1). Thus for $\alpha$ and $\beta$ in $\Gamma_{1}, \alpha<\beta$ if and only if $G_{\alpha}$ is a proper subset of $G_{\beta}$, and $\alpha=\beta$ if and only if $G_{\alpha}=G_{\beta}$. Also it is easy to show that a proper $l$-ideal of $G$ is regular if and only if it is meet irreducible in the lattice of all $l$-ideals of $G$.

Lemma 4.2. $V\left(\Gamma_{1}, G^{\gamma} / G_{\gamma}\right)$ is an l-group, and $\Gamma_{1}$ is a plenary subset of $\Gamma$.

Proof. By Theorem 4.1 for each $\gamma \in \Gamma_{1}, G / G_{\gamma}$ is an $o$-group, and hence the elements of $\Gamma$ that exceed $\gamma$ form a chain. Thus $\Gamma_{1}$ is a root system, and so by Theorem 2.2, $V\left(\Gamma_{1}, G^{\gamma} / G_{\gamma}\right)$ is an l-group.

If $g$ is a nonzero element of $G$, then by Zorn's Lemma there exists an $l$-ideal $C$ of $G$ that is maximal without $g$, and by Theorem 4.1 there exists a unique $l$-ideal $C^{\prime}$ of $G$ that covers $C$. Thus $\left(C^{\prime}, C\right)=\left(G^{\gamma}, G_{\gamma}\right)$ for some $\gamma \in \Gamma_{1}$. Therefore each nonzero element of $G$ has at least one value in $\Gamma_{1}$.

If $g \in G \backslash G^{\gamma}$ for some $\gamma \in \Gamma_{1}$, then there exists an $l$-ideal $M$ of $G$ that is maximal without $g$ and which contains $G^{\gamma}$. As before $M=G_{\delta}$ for some $\delta \in \Gamma_{1}$, and so $\delta>\gamma$ and $g \in G^{\delta} \mid G_{\delta}$. Therefore $\Gamma_{1}$ is a plenary subset of $\Gamma$.

Let

$$
\begin{aligned}
& \Gamma_{2}=\left\{\gamma \in \Gamma \mid G / G_{\gamma} \text { is an o-group }\right\}, \\
& \Gamma_{3}=\left\{\gamma \in \Gamma \mid \text { the } l \text {-ideals of } G \text { that contain } G_{\gamma} \text { form a chain }\right\} .
\end{aligned}
$$

Lemma 4.3. $\Gamma_{1}=\Gamma_{2}=\Gamma_{3} \subseteq \Lambda$.

Proof. By Theorem 4.1 $\Gamma_{1} \subseteq \Gamma_{2}$, and clearly $\Gamma_{2} \subseteq \Gamma_{3} \subseteq \Lambda$. Consider $G_{\gamma}$ for $\gamma \in \Gamma_{3}$, and let $g \in G^{\gamma} \mid G_{\gamma}$. Let $Q$ be an $l$-ideal of $G$ that contains $G_{\gamma}$ and which is maximal with respect to not containing $g$. Then $g \in G^{\gamma} \mid Q$, and since the $l$-ideals of $G$ that contain $G_{\gamma}$ form a chain, $G^{\gamma} \supset Q \supseteq G_{\gamma}$. However $G^{\gamma}$ covers $G_{\gamma}$, and so $Q=G_{\gamma \gamma}$ Thus $G_{\gamma}$ is regular, and hence $\Gamma_{3} \subseteq \Gamma_{1}$.

CoRollary. If $g \in G^{\gamma} \mid G_{\gamma}$ for $\gamma \in \Gamma_{1}$, then $G_{\gamma}$ is a maximal l-ideal of $G$ without $g$.

If $G / C$ is an $o$-group, where $C$ is an $l$-ideal of $G$, then $C=G_{\gamma}$ for some $\gamma \in \Gamma_{1}$ if and only if $C$ is covered by an $l$-ideal. Such a $C$ is not necessarily covered. 
There is a rather nice characterization of the plenary subsets of $\Gamma_{1}$.

LEMma 4.4. For a subset $\Delta$ of $\Gamma_{1}$ the following are equivalent:

(1) $\Delta$ is a plenary subset of $\Gamma_{1}$.

(2) $\Delta$ is a dual ideal of $\Gamma$ and $\bigcap_{\delta \in \Delta} G_{\delta}=\{0\}$.

Proof. Suppose that $\Delta$ is a plenary subset of $\Gamma_{1}$, and suppose that $\delta<\gamma$, where $\delta \in \Delta$ and $\gamma \in \Gamma$. If $g \in G^{\gamma} \backslash G_{\gamma}$, then $g \notin G^{\delta}$, and hence there exists $\delta^{\prime} \in \Delta$ such that $\delta^{\prime}>\delta$ and such that $g \in G^{\delta^{\prime}} \mid G_{\delta^{\prime}}$. Since the elements in $\Gamma$ that exceed $\delta$ are linearly ordered it follows that $\gamma=\delta^{\prime} \in \Delta$. Thus $\Delta$ is a dual ideal of $\Gamma$. If $g$ is a nonzero element of $G$, then there exists $\delta \in \Delta$ such that $g \in G^{\delta} \mid G_{\delta}$. Therefore $\bigcap_{\delta \in \Delta} G_{\delta}=\{0\}$.

Conversely suppose that $\Delta$ is a dual ideal of $\Gamma$ and that $\bigcap_{\delta \in \Delta} G_{\delta}=\{0\}$. If $\delta \in \Delta$ and if $g \notin G^{\delta}$, then there exists $\gamma \in \Gamma$ such that $\gamma>\delta$ and $g \in G^{\gamma} \mid G_{\gamma}$. But since $\Delta$ is a dual ideal of $\Gamma, \gamma \in \Delta$. If $g$ is a nonzero element of $G$, then there exists $\delta \in \Delta$ such that $g \notin G_{\delta}$ since $\bigcap_{\delta \in \Delta} G_{\delta}=\{0\}$. Either $g \in G^{\delta} \backslash G_{\delta}$, or as above there exists $\gamma>\delta$ such that $g \in G^{\gamma} \mid G_{\gamma}$ and $\gamma \in \Delta$. Therefore $\Delta$ is a plenary subset of $\Gamma_{1}$.

Let $\mathscr{C}$ be the collection of all $l$-ideals of $G$. For each nonzero element $g$ of $G$ there exists a subset $\mathscr{C}_{g}$ of $\mathscr{C}$ whose elements are those $l$-ideals which are maximal with respect to not containing $g$.

Throughout the remainder of this section, let $\Delta$ be a plenary subset of $\Gamma_{1}=\Gamma_{2}$. Note that $\Delta$ satisfies condition (b) of Theorem 2.2 , and hence $V\left(\Delta, G^{\delta} / G_{\delta}\right)$ is an l-group. This is precisely why we are interested in $\Gamma_{2}$.

LEMMA 4.5. If $0<x$ and $0 \leqq y$ are disjoint elements of $G$ (i.e., $x \wedge y=0$ ) and if $C \in \mathscr{C}_{x}$, then $y \in C$ and no l-ideal of $G$ which is maximal without $y$ is contained in $C$.

Proof. Since the lemma is obvious if $y=0$, we assume that $0<y$. If $y \notin C$, then $x+C$ and $y+C$ are positive disjoint elements in the $o$-group $G / C$ (Theorem 4.1), and this is impossible. If $K \in \mathscr{C}_{y}$, then $x \in K$, and hence $K \nsubseteq C$.

LEMMA 4.6. Let $g$ be a nonzero element in $G$. Then

(i) Each value of $g \vee 0$ in $\Gamma_{1}$ is also a value of $g$.

(ii) If $\delta \in \Gamma_{1}$ is a value of $g$ and $g+G_{\delta}$ is negative in $G^{\delta} / G_{\delta}$, then $g \vee 0 \in G_{\alpha}$ for all $\alpha \leqq \delta\left(\alpha \in \Gamma_{1}\right)$.

(iii) Let $\Lambda$ be any subset of $\Gamma_{1}$ that contains at least one value for every element in $G$. Then $g$ is positive if and only if $g+G_{\delta}$ is positive in $G^{\delta} / G_{\delta}$ for all values $\delta$ of $g$ in $\Lambda$.

Proof. (i) If $g^{+}=g \vee 0 \in G^{\delta} \mid G_{\delta}$, then, by Lemma 4.5, $g^{-}=-g \vee 0 \in G_{\delta}$, and hence $g=g^{+}-g^{-} \in G^{\delta} \backslash G_{\delta}$.

(ii) Suppose that $g \in G^{\delta} \backslash G_{\delta}$ and that $g+G_{\delta}$ is negative in $G^{\delta} / G_{\delta}$. Then $g-c<0$ for some $c \in G_{\delta}$, and thus $0 \leqq g \vee 0 \leqq c \vee 0 \in G_{\delta}$. Thus, $g \vee 0 \in G_{\delta}$. Suppose (by 
way of contradiction) that $g \vee 0 \notin G_{\alpha}$ for some $\alpha<\delta$. Then there exists a value $\beta \geqq \alpha$ of $g \vee 0$. By (i), $\beta$ is also a value of $g$, and since $g \vee 0 \in G_{\delta}, \beta \neq \delta$. Since the values of $g$ form a trivially ordered set, $\beta \| \delta$. Therefore $\alpha<\beta, \alpha<\delta$, and this is impossible in $\Gamma_{1}=\Gamma_{2}$.

(iii) If $0<g \in G^{\delta} \mid G_{\delta}$, then clearly $g+G_{\delta}$ is positive in the $o$-group $G^{\delta} / G_{\delta}$. If $0 \$ g$, then $0<g^{-}$. Let $\delta$ be a value of $g^{-}$in $\Lambda$. By (i) $\delta$ is also a value of $-g$, and hence a value of $g$. And by Lemma $4.5, g^{+} \in G_{\delta}$. Therefore $g+G_{\delta}=-g^{-}+G_{\delta}$ is negative.

Corollary. A subset $\Delta$ of $\Gamma_{1}$ is plenary if and only if

$(\alpha)$ each $0<g \in G$ has at least one value in $\Delta$, and

( $\beta)$ if $0<g \in G \mid G^{\delta}(\delta \in \Delta)$, then $g$ has a value in $\Delta$ that exceeds $\delta$.

This is an immediate consequence of our definition of plenary and part (i) of the lemma. Also note that it follows from this lemma that every $v$-isomorphism $\phi$ of $G$ into $V\left(\Delta, G^{\delta} / G_{\delta}\right)$ is an $o$-isomorphism (both $\phi$ and $\phi^{-1}$ preserve order).

If $G$ and $H$ are $l$-groups and if $\phi$ is an isomorphism which maps $G$ into $H$ and has the further property that $(x \vee y) \phi=x \phi \vee y \phi$ and $(x \wedge y) \phi=x \phi \wedge y \phi$ for all $x$ and $y$ in $g$, then $\phi$ is called an l-isomorphism.

LEMMA 4.7. If $\phi$ is a v-isomorphism of $G$ into $V\left(\Delta, G^{\delta} / G_{\delta}\right)$, then $\phi$ is an l-isomorphism. In particular, $G \phi$ is a sublattice of $V$.

Proof. It suffices to show that $(a \vee 0) \phi=a \phi \vee \theta$ for all $a \in G$. Let $M$ be a maximal chain of $\Delta$ and let $\pi$ be the projection of $V$ onto the $o$-group.

$$
V_{M}=\left\{v \in V \mid v_{\delta}=0 \text { for all } \delta \in \Delta \mid M\right\} \text {. }
$$

It suffices to show that $(a \vee 0) \phi \pi=a \phi \pi \vee \theta$ (see the description of $a \phi \vee \theta$ given in the proof of Theorem 2.2).

CASE I. $a \phi \pi \leqq \theta$. If $a \phi \pi=\theta$, then $(a \phi)_{\delta}=G_{\delta}$ for all $\delta \in M$; hence $a \in G_{\delta}$ for all $\delta \in M$, and so $a \vee 0 \in G_{\delta}$ for all $\delta \in M$. Thus $(a \vee 0) \phi \pi=\theta=a \phi \pi \vee \theta$. If $a \phi \pi<\theta$, then let $\delta$ be a value of $a$ in $M$. Then $a+G_{\delta}$ is negative, and hence by Lemma 4.6, $a \vee 0 \in G_{\alpha}$ for all $\alpha \in M$. Therefore, $(a \vee 0) \phi \pi=\theta=a \phi \pi \vee \theta$.

CASE II. $\theta<a \phi \pi$. Then $-a \phi \pi<\theta$, and hence by the above, $(-a \vee 0) \phi \pi=\theta$. Therefore

$$
(a \vee 0) \phi \pi=(a+(-a \vee 0)) \phi \pi=a \phi \pi+(-a \vee 0) \phi \pi=a \phi \pi=a \phi \pi \vee \theta .
$$

THEOREM 4.2 (MAIN EMBEDDING THEOREM FOR $l$-GROUPS). Let $G$ be a divisible, abelian l-group, and let $\Delta$ be a plenary subset of $\Gamma_{1}=\Gamma_{2}$. Then $V\left(\Delta, G^{\delta} / G_{\delta}\right)$ is an l-group and there exists a v-isomorphism $\phi$ of $G$ into $V$. Also $\phi$ is an $l$-isomorphism, and hence $G \phi$ is a sublattice of $V$.

This theorem is an immediate consequence of Theorems 2.2 and 3.1 and Lemma 4.7. The next theorem justifies our choice of $\Gamma_{1}$. 
THeOREM 4.3. Suppose that $G$ is an abelian l-group, $\Delta$ is a subset of $\Gamma$ and that $V\left(\Delta, G^{\delta} / G_{\delta}\right)$ is an l-group. If there exists a v-isomorphism of $G$ into $V$ which is also an l-isomorphism, then $\Delta$ is a plenary subset of $\Gamma_{1}$.

Proof. Let $\phi$ be a $v$-isomorphism of $G$ into $V$ that is also an $l$-isomorphism. It follows from Theorem 3.1 that $\Delta$ is a plenary subset of $\Gamma$ (divisibility of $G$ was not used in this part of the proof of Theorem 3.1). For each $\delta \in \Delta, G_{\delta} \phi=V_{\delta} \cap G$ where

$$
V_{\delta}=\left\{v \in V \mid v_{\alpha}=G_{\alpha} \text { for all } \alpha \geqq \delta\right\} .
$$

By a straightforward computation it follows that $\phi$ induces an $o$-isomorphism of $G / G_{\delta}$ into $V / V_{\delta}$. Let $v$ be an element in $V$ with a nonzero $\delta$ th component and all other components zero. Then (see the proof of Theorem 6.1) $V_{\delta}$ is a maximal $l$-ideal of $V$ without $v$, and hence by Theorem $4.1, V / V_{\delta}$ is an $o$-group. Therefore $G / G_{\delta}$ is an $o$-group, and hence for each $g \in G^{\delta} \backslash G_{\delta}, G_{\delta}$ is a maximal $l$-ideal of $G$ without $g$. Thus $\delta \in \Gamma_{1}$ and hence $\Delta \subseteq \Gamma_{1}$.

Note that $\Gamma_{1}$ may contain many plenary subsets and for each such subset we get an embedding. In order to obtain the best possible embedding of this type one needs to know when $\Gamma_{1}$ admits a minimal plenary subset. The next section is devoted to answering this question. Theorem 5.2 asserts that when such a minimal set exists it is unique, and in Theorem 5.4 we find a property of $G$ that is both necessary and sufficient for the existence of a minimal plenary subset of $\Gamma_{1}$.

Note that if $\Delta$ is a plenary subset of $\Gamma_{1}$, and $\phi$ is a $v$-isomorphism of $G$ into $V\left(\Delta, G^{\delta} / G_{\delta}\right)$ such that $G \phi$ contains the small direct sum of the $G^{\delta} / G_{\delta}$, then $\Delta$ is a minimal plenary subset of $\Gamma_{1}$. For if $\delta \in \Delta$, then there exists an element $g \in G$ whose only value in $\Delta$ is $\delta$. Thus $\delta$ cannot be discarded, and hence $\Delta$ is minimal.

Finally, we note that the Lorenzen embedding can be recovered from the material in this section. For let $\Lambda$ be any subset of $\Gamma_{1}$ such that every nonzero element of $G$ has at least one value in $\Lambda$. By Theorem $4.1, G / G_{\gamma}$ is an $o$-group for each $\gamma \in \Lambda$. Let $\pi_{\gamma}$ be the natural homomorphism of $G$ onto $G / G_{\gamma}$ for each $\gamma \in \Lambda$, and let $L=L\left(\Lambda, G / G_{\gamma}\right)$ be the large cardinal sum of the $G / G_{\gamma}(\gamma \in \Lambda)$. The mapping $\pi$ of $g \in G$ upon $\left(\cdots, g \pi_{y}, \cdots\right) \in L$ is clearly an isomorphism. By (iii) of Lemma 4.6 , it follows that both $\pi$ and $\pi^{-1}$ preserve order. If $\theta$ is the identity for $L$, and $g \in G$, then

$$
\begin{aligned}
g \pi \vee \theta & =\left(\cdots, g+G_{\gamma}, \cdots\right) \vee \theta \\
& =\left(\cdots,(g \vee 0)+G_{\gamma}, \cdots\right) \\
& =(g \vee 0) \pi .
\end{aligned}
$$

Therefore $G \pi$ is a sublattice of $L$, and clearly $G \pi$ is a subdirect sum of $L$.

In general, this embedding does not fill out $L$ very nicely. In particular, if $G$ is a non-Archimedean o-group, then $L$ is not an o-group. In $\$ 6$, we make use of Theorem 4.2 to obtain a much better Lorenzen embedding (for divisible, abelian $l$-groups). 
5. Minimal plenary sets. Throughout this section let $G$ be an abelian l-group, and let $\Gamma_{1}$ be as in $\$ 4$. As in $\$ 3$ we denote the two properties of a plenary set by $(\alpha)$ and $(\beta)$.

Let $W$ be a partially ordered set, and let $w \in W$. Then the set $\{x \in W \mid x \leqq w\}$ is called the cone beneath $w$. Since each plenary subset of $\Gamma_{1}$ is a dual ideal of $\Gamma$ (Lemma 4.4) we have the following:

Lemma 5.1. Let $\Delta$ be a plenary subset of $\Gamma_{1}$, and let $\delta \in \Delta$. If $\delta<\gamma \in \Gamma_{1}$, then $\gamma \in \Delta$. Thus, if we wish to discard an element $\gamma$ in $\Gamma_{1}$, and retain a plenary subset of $\Gamma_{1}$, the cone beneath $\gamma$ in $\Gamma_{1}$ must also be discarded.

THEOREM 5.1. Let $\Delta$ be a plenary subset of $\Gamma_{1}$. Then the following conditions are equivalent:

(1) $\Delta$ is a minimal plenary subset of $\Gamma_{1}$.

(2) For each $\delta \in \Delta$ there exists $g \in G$ such that all the values of $g$ in $\Delta$ are less than or equal to $\delta$.

(3) For each $\delta \in \Delta$ there exists $g \in G$ such that all the values of $g$ in $\Gamma_{1}$ are less than or equal to $\delta$.

Proof. First suppose that $\Delta$ is a minimal plenary subset of $\Gamma_{1}$. And assume, by way of contradiction, that there exists $\delta \in \Delta$ such that no nonzero element of $G$ has all of its values less than or equal to $\delta$. Let $\Delta^{\prime}$ be the subset of $\Delta$ obtained by discarding $\delta$ and the cone beneath it. Then each nonzero element $g$ of $G$ has a value in $\Delta^{\prime}$, and clearly $\Delta^{\prime}$ satisfies $(\beta)$. Thus $\Delta^{\prime}$ is plenary, and this contradicts the minimality of $\Delta$. Therefore condition (1) implies condition (2).

Next assume that condition (2) holds, and suppose, by way of contradiction, that $\delta \in \Delta$ and that no element of $G$ has all of its values in $\Gamma_{1}$ beneath $\delta$. Then there exists a nonzero element $g$ of $G$ such that all of the values of $g$ in $\Delta$ are beneath $\delta$. It follows that if $\gamma \in \Delta$ and $\gamma \neq \delta$, then $g \in G_{\gamma}$. By Lemma 4.6 we may assume that $g>0$. By assumption, $g$ has a value $\beta \neq \delta$ where $\beta \in \Gamma_{1} \backslash \Delta$. Select $0>f \in G_{\beta} \backslash G_{\delta}$. If $\delta$ is a value of $f$, then by the Archimedean property of $G^{\delta} / G_{\delta}$, for some positive integer $n, n f+g+G_{\delta}$ is negative. Thus we may assume without loss of generality that if $\delta$ is a value of $f$, then $\delta$ is a value of $f+g$ and $f+g+G_{\delta}$ is negative. Let $h=f+g$. Since $f \notin G_{\delta}, f$ has a value $\delta^{\prime} \geqq \delta, \delta^{\prime} \in \Delta$. If $\delta=\delta^{\prime}$, then $G_{\delta}+h$ is negative and $\delta$ is a value of $h$. If $\delta^{\prime}>\delta$, then $g \in G_{\delta^{\prime}}$, and so $\delta^{\prime}$ is a value of $h$ and $G_{\delta^{\prime}}+h=G_{\delta^{\prime}}+f$ is negative.

Now let $\gamma$ be a value of $h$ in $\Delta$. If $\gamma \leqq \delta$, then $\gamma=\delta=\delta^{\prime}$, and therefore by the above $h+G_{\gamma}$ is negative. If $\gamma \neq \delta$, then $g \in G_{\gamma}$ and so $G_{\gamma}+h=G_{y}+f$ is negative. However $\beta$ is a value of $h$, and $G_{\beta}+h=G_{\beta}+g$ is positive since $g>0$ and $0>f \in G_{\beta}$. This contradicts Lemma 4.6, and proves that condition (2) implies condition (3).

Lastly assume that condition (3) is true. By Lemma 5.1, in order to get a proper plenary subset of $\Delta$, we must discard a cone beneath some element of $\Delta$. Clearly, since $\Delta$ satisfie; condition (3), all values of some nonzero element $g$ of $G$ must 
lie in that cone. Thus, since we need to retain property $(\alpha)$, no cone can be discarded, and hence $\Delta$ is a minimal plenary set.

An element $\delta \in \Gamma_{1}$ is called essential if there is an $h \in G$ such that all of the values of $h$ are in the cone beneath $\delta$. Let $E$ denote the set of all essential elements of $\Gamma_{1}$.

THEOREM 5.2. If $\Delta$ is a minimal plenary subset of $\Gamma_{1}$, then $\Delta=E$. Thus if $\Gamma_{1}$ contains a minimal plenary subset, that subset is unique.

For each nonzero element $g$ of $G$ let $R_{g}$ be the subgroup of $G$ that is generated by the $l$-ideals of $G$ that do not contain $g$. Then $R_{g}$ is an $l$-ideal, and therefore, it is the intersection of all the $l$-ideals of $G$ that contain all of the $G_{\gamma}$, where $\gamma$ is a value of $g$ in $\Gamma_{1}$. Let

$$
R(G)=\bigcap\left\{R_{g} \mid 0 \neq g \in G\right\} .
$$

The $l$-ideal $R(G)$ can be characterized in another way.

THEOREM 5.3. If $g$ is a nonzero element of $G$, then $g \in R(G)$ if and only if $g$ has no value which is essential.

Proof. If $g \notin R(G)$ then there exists $0 \neq h \in G$ such that $g \notin R_{h}$. There is an $l$-ideal $G_{\alpha}$ maximal without $g$ such that $G_{\alpha} \supseteq R_{h}$. Then $\alpha$ is a value of $g$, and all values of $h$ are in the cone beneath $\alpha$. Hence $\alpha$ is essential. Conversely if $\alpha$ is an essential value of $g$ then there exists $0 \neq h \in G$ such that all the values of $h$ are in the cone under $\alpha$. Therefore $g \notin G_{\alpha} \supseteq R_{h}$. Hence $g \notin R(G)$.

As an immediate consequence of Theorem 5.3 and Theorem 5.2 we have

THeOrem 5.4. A minimal plenary subset of $\Gamma_{1}$ exists if and only if $R(G)=0$.

REMARKS. If $G=R(G)$ then it follows from Lemma 5.4 that each $0 \neq g \in G$ has an infinite number of values. An example of such a group is the $l$-group of all real valued continuous functions on the unit interval $[0,1]$. Also it can be shown that if $S$ is an $l$-ideal of $G$, then $R(S)=S \cap R(G)$. Thus it follows that $R(R(G))$ $=R(G)$; and if $A$ and $B$ are $l$-ideals of $G$, then $R(A \cap B)=R(A) \cap R(B)$ and $R(A+B)=R(A)+R(B)$.

An element $g$ of $G$ is special if it is positive and has a unique value $\delta$ in $\Gamma_{1}$. A value of a special element of $G$ is called a special element of $\Gamma_{1}$. Clearly any plenary subset of $\Gamma_{1}$ must contain all the special elements of $\Gamma_{1}$.

LEMMA 5.2. Let $Q$ be a set of special elements in $G$, and let $\Delta$ be the set of values for the elements in $Q$. Let

$$
\Delta^{\prime}=\left\{\alpha \in \Gamma_{1} \mid \alpha \geqq \delta \text { for some } \delta \in \Delta\right\} \text {. }
$$

If each positive element in $G$ is greater than or equal to at least one element of $Q$, then $\Delta^{\prime}$ is the unique minimal plenary subset of $\Gamma_{1}$, and 


$$
\Delta^{\prime}=\left\{\alpha \in \Gamma_{1} \mid \alpha \geqq \delta \text { for some special } \delta \in \Gamma_{1}\right\} \text {. }
$$

Proof. It follows from Lemma 5.1 and the remarks preceding this lemma that $\Delta^{\prime}$ must be contained in any plenary subset of $\Gamma_{1}$, and clearly $\Delta^{\prime}$ satisfies $(\beta)$. Thus it suffices by the corollary to Lemma 4.6 to show that every $0<g \in G$ has at least one value in $\Delta^{\prime}$. There exists $q \in Q$ such that $0<q<g$. Let $\delta$ be the value of $q$. Since $G_{\delta}$ is convex and $q \notin G_{\delta}, g \notin G_{\delta}$. Thus $g$ has a value $\alpha \geqq \delta\left(\alpha \in \Gamma_{1}\right)$, and since $\delta \in \Delta^{\prime}, \alpha \in \Delta^{\prime}$.

An element $g$ of $G$ is said to be basic if $0<g$ and $\{x \in G \mid 0<x \leqq g\}$ is totally ordered. A subset $S$ of $G$ is a basis for $G$ if $S$ is a maximal set of disjoint elements and each $s$ in $S$ is basic (see [6] for the theory of basic elements and bases).

Lemma 5.3. Each basic element of $G$ is special. Let $g$ be a basic element in $G$, and let $U$ be the maximal l-ideal of $G$ without $g$. Let

$$
\mathscr{N}=\left\{L \mid L \subset U \text { and } L \in \mathscr{C}_{h} \text { for some } 0 \neq h \in G\right\} .
$$

Then each element of $\mathscr{N}$ is determined by a basic element that is less than $g$, and hence $\mathscr{N}$ is totally ordered.

Proof. Let $a$ be a basic element of $G$. Then $0<a$, and there exists a maximal convex $o$-subgroup $G^{\prime}$ of $G$ that contains $a$ [6, Lemma 2.1]. Let $A$ and $B$ be the pair of convex $o$-subgroups of $G^{\prime}$ such that $A$ covers $B$ and $a \in A \backslash B$.

CASE I. $B=0$. Since $\left\{x \in G^{+} \mid x \wedge a=0\right\}$ is a convex subsemigroup of positive elements $[3$, Theorem 6, p. 219], it follows that

$$
U=\left[\left\{x \in G^{+} \mid x \wedge a=0\right\}\right]
$$

is an $l$-ideal of $G$ that does not contain $a[6$, p. 214]. Let $V$ be any $l$-ideal of $G$ that does not contain $a$. If $0<v \in V \backslash U$, then $0<v \wedge a \in A \cap V$. Thus, since $A$ is an Archimedean $o$-group, $n(v \wedge a)>a>0$, for some positive integer $n$. But this means that $a \in V$, a contradiction. Therefore $V \subseteq U$, and hence $U$ is the unique maximal $l$-ideal of $G$ without $a$.

CASE II. $B \neq 0$. Let $B^{*}=\left\{x \in G^{+} \mid x \wedge B^{+}=0\right\}$. Then $B^{+}+B^{*}=\left\{x \in G^{+} \mid x\right.$ does not exceed every element of $B\}$ and

$$
U=\left[B^{+}+B^{*}\right]
$$

is an $l$-ideal of $G$ which does not contain $a[6$, p. 223].

If $g \in G^{+} \mid U$, then $g>B$ and $a>B$, and hence $g \wedge a \geqq B$; but since $B$ has no largest element, $g \wedge a>B$. Since $A / B$ is an Archimedean $o$-group, it follows that $n(g \wedge a)>a>0$ for some integer $n>0$. Thus any $l$-ideal of $G$ without $a$ must be contained in $U$. Therefore $U$ is the unique maximal $l$-ideal of $G$ without $a$.

Thus $a$ is special. Suppose that $V$ is a maximal $l$-ideal of $G$ without $v>0$ and $V \subseteq U$, where $U$ is the maximal $l$-ideal without the basic element $a$. If $v \wedge a=0$, then by Lemma 4.5, $a \in V \subseteq U$, a contradiction. Therefore $0<v \wedge a \leqq v$. If 
$v \wedge a \notin V$, then $V$ is maximal without $v \wedge a$, and since $v \wedge a \leqq a$ it is basic. Finally, suppose that $v \wedge a \in V$. Then $v=v \wedge a+v^{\prime}$, where $0<v^{\prime}<v$ and $v^{\prime} \notin V$. Then $V$ is maximal without $v^{\prime}$. Also, $a=v \wedge a+a^{\prime}$, and $a^{\prime} \wedge v^{\prime}=0$. If $a^{\prime}=0$, then $a \leqq v$, and hence $V=U$. If $a^{\prime} \neq 0$, then, since $a^{\prime}$ and $a \wedge v^{\prime}$ are elements of the $o$-group $A$, we have that $0<a \wedge v^{\prime}$ implies $0<a^{\prime} \wedge v^{\prime}$, a contradiction. Thus, if $a^{\prime} \neq 0$, then $a \wedge v^{\prime}=0$, and by Lemma 4.5, $a \in V$, a contradiction. Thus $V$ is determined by a basic element that is less than or equal to $a$, and since such basic elements are linearly ordered, $\mathscr{N}$ must be linearly ordered.

If $G$ has a basis, then each positive element of $G$ is greater than or equal to at least one basic element [6, Theorem 5.1]. Thus the following theorem is an immediate consequence of Lemmas 5.2 and 5.3.

THEOREM 5.5. Suppose that $G$ is an abelian l-group with a basis $Q$. Let $\Delta$ be the set of values in $\Gamma_{1}$ for the elements in $Q$, and let

$$
\Delta^{\prime}=\left\{\alpha \in \Gamma_{1} \mid \alpha \geqq \delta \text { for some } \delta \in \Delta\right\} .
$$

Then $\Delta^{\prime}$ is the minimal plenary subset of $\Gamma_{1}$.

THEOREM 5.6. If an abelian l-group $G$ has a basis, then there exists a minimal plenary subset $\Delta$ of $\Gamma_{1}$ which satisfies

(*) for each $\alpha \in \Delta$ there exists a $\beta \in \Delta$ such that $\alpha \geqq \beta$ and the elements in $\Delta$ below $\beta$ form a chain. Conversely, if $\Delta$ is a minimal plenary subset of $\Gamma_{1}$ which satisfies (*), then $G$ has a basis.

Proof. If $G$ has a basis, then the result follows immediately from Lemma 5.3 and Theorem 5.5.

Conversely, suppose that $\Delta$ is a minimal plenary subset of $G$ which satisfies (*). Let $\delta$ be an element in $\Delta$ with no incomparable elements of $\Delta$ below it. Since $\Delta$ is minimal, it follows from Theorem 5.2 that there exists a special element $g>0$ with value $\gamma \leqq \delta$. Assume (by way of contradiction) that $g$ is not basic. Then there exist positive elements $a$ and $b$ of $G$ that are less than $g$ and incomparable. If $a \wedge b \neq 0$, then let $a^{\prime}=a-(a \wedge b)$ and $b^{\prime}=b-(a \wedge b)$. It follows that $a^{\prime}$ and $b^{\prime}$ are positive, disjoint, and less than $g$. Thus, without loss of generality, we may assume that $a \wedge b=0$. Let $\alpha(\beta)$ be a value of $a(b)$ in $\Delta$. Since $0<a<g$, $g \notin G_{\alpha}$, and hence $\alpha \leqq \gamma$. Similarly, $\beta \leqq \gamma$. But by Lemma $4.5, a \in G_{\beta} \mid G_{\alpha}$ and $b \in G_{\alpha} \mid G_{\beta}$, and hence by Lemma 4.4, $\alpha \| \beta$, a contradiction. Therefore, $g$ is basic.

Now it suffices [6, Theorem 5.1] to show that every positive element of $G$ is greater than or equal to at least one basic element. Let $h$ be a positive element in $G$ with value $\delta \in \Delta$. By the above argument, there exists a basic element $g$ with value $\gamma \leqq \delta$. If $h \wedge g=0$, then by Lemma $4.5, h \in G_{\gamma} \subseteq G_{\delta}$. But $h \in G^{\delta} \backslash G_{\delta}$; thus $0<h \wedge g \leqq h$. Hence $h$ is greater than or equal to the basic element $h \wedge g$.

THEOREM 5.7. If $G$ is an Archimedean l-group, then $\Gamma_{1}$ contains a minimal plenary subset if and only if $G$ has a basis. 
Proof. By Theorem 5.6, if $G$ has a basis, then $\Gamma_{1}$ contains a unique minimal plenary subset.

Conversely, suppose that $\Gamma_{1}$ contains a minimal plenary subset $\Delta$. It suffices to show that $\Delta$ is trivially ordered. For then $\Delta$ clearly satisfies property (*) of Theorem 5.6, and hence $G$ has a basis.

Suppose that $\alpha$ and $\beta$ are elements of $\Delta$ and that $\alpha<\beta$. By Theorem 5.1, there exists an element $g$ of $G$, all of whose values are less than or equal to $\alpha$. By Lemma 4.6, we may assume that $0<g$. If $0<h \in G^{\beta} \backslash G_{\beta}$, then $n g<h$ for all integers $n>0$, since the values of $n g$ are all less than or equal to $\alpha$. Thus, since $G$ is Archimedean, $g \leqq 0$, a contradiction. Therefore, $\alpha \| \beta$ for all $\alpha$ and $\beta$ in $\Delta$.

As before, let $G$ be an abelian $l$-group, and let $\Delta$ be a plenary subset of $\Gamma_{1}$. The rest of the theorems in this section follow from the preceding theory and the following lemma.

LEMma 5.4. If $0<g \in G$ has only a finite number of values $\delta_{1}, \delta_{2}, \cdots, \delta$ in $\Delta$, then there exists an element $h$ in $G$ such that $\delta_{1}$ is the only value of $h$ in $\Delta$ and $g+G_{\delta_{1}}=h+G_{\delta_{1}}$.

Proof. The proof is by induction on the number of values of $g$. Clearly, if $n=1$, then the lemma is true.

Suppose that the lemma is true for all positive elements of $G$ which have less than $n$ values in $\Delta$ and that $0<g \in G$ has $n$ values $\delta_{1}, \cdots, \delta_{n}$ in $\Delta$. The set of values of $g$ forms a trivially ordered set, and therefore $G_{\delta_{1}} \| G_{\delta_{2}}$. Thus there exists $0>k \in G_{\delta_{1}} \mid G_{\delta_{2}}$. In particular, $m k+g \in G^{\delta_{1}} \mid G_{\delta_{1}}$, and $g+G_{\delta_{1}}=m k+g+G_{\delta_{1}}=$ $((m k+g) \vee 0)+G_{\delta_{1}}$ for all integers $m>0$. Let $Q=\left\{\alpha \in \Delta\left|k \in G^{\alpha}\right| G_{\alpha}\right.$ and $\alpha \geqq \delta_{i}$ for some $i=1, \cdots, n\}$. Then $Q \neq \square$ because $k \notin G_{\delta_{2}}$, and since $Q \subseteq \Gamma_{2}, Q$ contains at most $n-1$ elements. If $\delta_{i}<\alpha \in Q$, then $k+g+G_{\alpha}$ is negative and if $\delta_{i} \in Q$, then $n k+g+G_{\delta_{i}}$ is negative for some $n>0$. Let $m$ be a positive integer such that $m k+g+G_{\alpha}$ is negative for all $\alpha$ in $Q$.

Now we claim that the only values of $(m k+g) \vee 0$ in $\Delta$ are contained in the set $\left\{\delta_{1}, \delta_{3}, \cdots, \delta_{n}\right\}$. If this is true, then, by induction, there exists an element $h$ of $G$ whose only value in $\Delta$ is $\delta_{1}$, and we are through.

The values of $(m k+g) \vee 0$ are contained in the values of $m k+g$ by Lemma 4.6, and if $\beta$ is a value of $m k+g$ and $m k+g+G_{\beta}$ is negative, then $(m k+g) \vee 0 \in G_{\alpha}$ for all $\alpha \leqq \beta$ by Lemma 4.6. Thus it suffices to prove that if $\alpha \in \Delta$ is a value of $m k+g$, such that $m k+g+G_{\alpha}$ is positive, then $\alpha=\delta_{i}$ for some $i=1,3, \cdots, n$.

If $\alpha<\delta_{i}$ for some $i$, then $m k+g+G_{\delta_{i}}=G_{\delta_{i}}$. Now $G / G_{\alpha}$ is an o-group, $G^{\delta_{i}} / G_{\alpha}$ is the smallest convex subgroup of $G / G_{\alpha}$ that contains $g+G_{\alpha}$, and $G^{\alpha} / G_{\alpha} \subset G^{\delta_{i}} / G_{\alpha}$. Therefore, $G^{\delta_{i}} / G_{\alpha}$ must be the smallest convex subgroup of $G / G_{\alpha}$ which contains $m k+G_{\alpha}$, and so, $\delta_{i}$ must be a value of $m k$. Hence, $\delta_{i} \in Q$, and $m k+g+G_{\delta_{i}}$ is negative, a contradiction. So no value of $m k+g$ can be less than a value of $g$. 
If $\delta_{2} \leqq \alpha$ or if $\delta_{i}<\alpha$ for some $i \neq 2$, then, by our choice of $m$ we know that $m k+g+G_{\delta_{i}}$ is negative, and hence $m k+g+G_{\alpha}$ is negative.

If $\alpha \| \delta_{i}$ for all $i$, then $g \in G_{\alpha}$, and hence $m k+g+G_{\alpha}=m k+G_{\alpha}$ is negative; this completes the proof of the lemma.

THEOREM 5.8. If for each $\delta \in \Delta$ there exists an element $g \in G^{\delta} \backslash G_{\delta}$ with only a finite number of values in $\Delta$, then $\Delta$ is the minimal plenary subset of $\Gamma_{1}$

Proof. By Lemma 5.4, for each $\delta \in \Delta$ there exists a $g \in G$ whose only value in $\Delta$ is $\delta$. Thus no proper subset of $\Delta$ satisfies condition $(\alpha)$ in the definition of a plenary subset.

Theorem 5.9. Suppose that $G$ is divisible and that each element in $\Delta$ is the value of some element $g$ in $G$ with only a finite number of values in $\Delta$. Then there exists a v-isomorphism $\phi$ of $G$ onto a sublattice of $V\left(\Delta, G^{\delta} / G_{\delta}\right)$ such that $G \phi$ contains the small direct sum of the $G^{\delta} / G_{\delta}$.

Proof. By Lemma 5.4, each element $\delta$ in $\Delta$ is the value of some element $g>0$ of $G$ with only one value in $\Delta$. Let $0<h \in G^{\delta} \mid G_{\delta}$. Without loss of generality we may assume that $g+G_{\delta} \geqq h+G_{\delta}$. Then $g \wedge h+G_{\delta}=h+G_{\delta}$, and for any $\beta \in \Delta$ such that $\beta \geq \delta, 0 \leqq g \wedge h \leqq g \in G_{\beta}$. Hence $\delta$ is the only value of $g \wedge h$ in $\Delta$. Thus any positive coset of $G^{\delta} / G_{\delta}$ contains an element whose only value in $\Delta$ is $\delta$. The existence of a $v$-isomorphism with the stated property now follows from Theorem 4.2 and the results in the appendix.

A subset $\Delta^{\prime}$ of $\Delta$ is said to be a lower subset if the relations $\alpha \in \Delta, \beta \in \Delta^{\prime}$, and $\alpha<\beta$ always imply that $\alpha \in \Delta^{\prime}$. For each $l$-ideal $I$ of $G$ let

$$
I \rho=\{\delta \in \Delta \mid \delta \leqq \text { some value in } \Delta \text { of an element } a \text { of } I\} .
$$

Clearly $I \rho$ is a lower subset of $\Delta$. Conversely for each lower subset $\Delta^{\prime}$ of $\Delta$ let

$$
\begin{aligned}
\Delta^{\prime} \rho^{\prime} & =\left\{g \in G \mid g \in G_{\alpha} \text { for all } \alpha \in \Delta \backslash \Delta^{\prime}\right\} \\
& =\left\{g \in G \mid \text { each value of } g \text { in } \Delta \text { belongs to } \Delta^{\prime}\right\} .
\end{aligned}
$$

Clearly $\Delta^{\prime} \rho^{\prime}$ is an $l$-ideal of $G$. If $\pi$ is a $v$-isomorphism of $G$ into $V\left(\Delta, G^{8} / G_{\delta}\right)$, then $\pi$ induces a $v$-isomorphism $\pi^{\prime}$ of $G / \Delta^{\prime} \rho^{\prime}$ into $V\left(\Delta \backslash \Delta^{\prime}, G^{\delta} / G_{\delta}\right)$; namely, for $g \in G$ and $\alpha \in \Delta \backslash \Delta^{\prime}$ define $\left(\left(g+\Delta^{\prime} \rho^{\prime}\right) \pi^{\prime}\right)_{\gamma}=(g \pi)_{\gamma}$

THEOREM 5.10. Suppose that each element $g$ in $G$ has at most a finite number of values in $\Delta$. Then $\rho$ is a one-to-one order preserving mapping of the set $\mathscr{I}$ of all l-ideals of $G$ onto the set $\mathscr{L}$ of all lower subsets of $\Delta$ and $\rho^{\prime}$ is the inverse of $\rho$.

Proof. It suffices to show that $\rho \rho^{\prime}$ and $\rho^{\prime} \rho$ are identity mappings. Let $\Delta^{\prime}$ be a lower subset of $\Delta$. If $\alpha \in \Delta^{\prime} \rho^{\prime} \rho$ then there exists an $a \in \Delta^{\prime} \rho^{\prime}$ such that $\alpha \leqq$ some value $\gamma$ in $\Delta$ of $a$. But all of the values of $a$ in $\Delta$ are elements of $\Delta^{\prime}$. Thus, since $\Delta^{\prime}$ is a lower subset of $\Delta$ and $\alpha \leqq \gamma \in \Delta^{\prime}, \alpha \in \Delta^{\prime}$. Therefore $\Delta^{\prime} \rho^{\prime} \rho \subseteq \Delta^{\prime}$. 
Consider $\alpha \in \Delta^{\prime}$. By Lemma 5.4 there exists an element $g \in G$ with $\alpha$ as its only value in $\Delta$. Thus $g \in \Delta^{\prime} \rho^{\prime}$, and hence $\alpha \in \Delta^{\prime} \rho^{\prime} \rho$. Therefore $\Delta^{\prime} \rho^{\prime} \rho \supseteq \Delta^{\prime}$.

Let $I$ be an $l$-ideal of $G$, and consider $a \in I$ and $\alpha \in \Delta \backslash I \rho$. If $a \notin G_{\alpha}$, then $a$ has a value in $\Delta$ that exceeds $\alpha$, and hence $\alpha \in I \rho$, a contradiction. Thus $a \in G_{\alpha}$, and it follows that $a \in I \rho \rho^{\prime}$. Therefore $I \subseteq I \rho \rho^{\prime}$.

Finally, consider $0<a \in I \rho \rho^{\prime}$, and let $\alpha_{1}, \cdots, \alpha_{n}$ be the values of $a$ in $\Delta$. Each $\alpha_{i} \in I \rho$, and hence $\alpha_{i} \leqq \delta_{i}$ where $\delta_{i} \in \Delta$ and $\delta_{i}$ is a value of an element $a_{i} \in I$. Without loss of generality we may choose the $a_{i}$ so that they are positive and so that

$$
a+G_{\alpha_{i}}<a_{i}+G_{\alpha_{i}}, \quad i=1, \cdots, n .
$$

Let $b=a_{1}+\cdots+a_{n}$. Then $0<a<b \in I$, and hence $a \in I$. Therefore $I \rho \rho^{\prime} \subseteq I$, and this completes the proof of the theorem.

Note that under the hypothesis that for each $\delta \in \Delta$ there exists an element $g \in G^{\delta} \backslash G_{\delta}$ with only a finite number of values in $\Delta, \Delta^{\prime} \rho^{\prime} \rho=\Delta^{\prime}$ and $I \subseteq I \rho \rho^{\prime}$ for all lower subsets $\Delta^{\prime}$ of $\Delta$ and for all $l$-ideals $I$ of $G$. It is only in proving $I \rho \rho^{\prime} \subseteq I$ that the stronger hypothesis was used.

THEOREM 5.11. If $\Delta$ contains only a finite number of maximal chains, then $\Delta=\Gamma_{1}$, and $G$ has a finite basis. If, in addition, $G$ is divisible, then there exists a v-isomorphism $\phi$ of $G$ onto a sublattice of $V\left(\Delta, G^{\delta} / G_{\delta}\right)$ such that $G \phi$ contains the small direct sum of the $G^{\delta} / G_{\delta}$. Conversely, if $G$ has a finite basis, consisting of exactly $n$ elements, then $\Gamma_{1}$ contains exactly $n$ maximal chains. In particular, if $G$ is divisible and if $\Gamma_{1}$ is finite, then $G \cong V\left(\Gamma_{1}, G^{\gamma} / G_{\gamma}\right)$.

Proof. The values of $0 \neq g \in G$ form a trivially ordered subset of $\Delta$, and hence they must lie in distinct maximal chains of $\Delta$. Thus if $\Delta$ contains only a finite number of such chains, then each element in $G$ has at most a finite number of values in $\Delta$. Therefore, by Theorem $5.8, \Delta$ is the minimal plenary subset of $\Gamma_{1}$, and the $v$-isomorphism $\phi$ described in Theorem 5.9 exists provided that $G$ is divisible.

Next suppose that the set $s_{1}, \cdots, s_{n}$ is a basis for $G$, and denote the value of $s_{i}$ by $\delta_{i}$ for $i=1, \cdots, n$. Let

$$
\Delta=\left\{\alpha \in \Gamma_{1} \mid \alpha \geqq \delta_{i} \text { for some } i=1, \cdots, n\right\} .
$$

By Lemma 5.3, the elements in $\Gamma_{1}$ that are less than or equal to $\delta_{i}(i=1, \cdots, n)$ form a chain, and each corresponds to a basic element. Let

$$
\Delta^{\prime}=\Delta \cup\left\{\alpha \in \Gamma_{1} \mid \alpha \leqq \delta_{i} \text { for some } i=1, \cdots, n\right\} .
$$

Then $\Delta^{\prime}$ contains exactly $n$ maximal chains, namely, the $n$ maximal chains that contain the $\delta_{i}(i=1, \cdots, n)$. Also, $\Delta=\left\{\alpha \in \Gamma_{1} \mid \alpha \geqq \delta\right.$ where $\delta$ is the value of some basic element $\}$. Therefore, by Theorem $5.5, \Delta^{\prime}$ is the minimal plenary subset of $\Gamma_{1}$. Suppose (by way of contradiction) that $\gamma \in \Gamma_{1} \mid \Delta^{\prime}$, and let $\Delta^{\prime \prime}$ be the subset of $\Gamma_{1}$ 
consisting of the elements of $\Delta^{\prime}, \gamma$, and the chain of all elements in $\Gamma_{1}$ that exceed $\gamma$. Clearly $\Delta^{\prime \prime}$ is a plenary subset of $\Gamma_{1}$, and $\Delta^{\prime \prime}$ contains $n+1$ maximal chains. Thus by the first part of the proof, $\Delta^{\prime \prime}$ is a minimal plenary subset of $\Gamma_{1}$, a contradiction. Therefore $\Gamma_{1}$ contains exactly $n$ maximal chains.

To complete the proof of this theorem, it suffices to show that if $\Delta$ contains only a finite number of maximal chains, then $G$ has a finite basis.

Let $a$ and $b$ be positive disjoint elements of $G$ with values $\alpha$ and $\beta$ respectively in $\Delta$. By Lemma $4.5, a \in G_{\beta} \mid G_{\alpha}$, and $b \in G_{\alpha} \mid G_{\beta}$. Thus, by Lemma $4.4, \alpha \| \beta$. It follows that $G$ does not contain an infinite disjoint set, and hence [6, Theorem 5.2], $G$ has a finite basis.

6. The groups $V\left(\Gamma, R_{\gamma}\right)$ and the Lorenzen embedding. Let $\Gamma$ be a root system ( $p o$-set in which no incomparable elements have a common lower bound), and for each $\gamma \in \Gamma$ let $R_{\gamma}$ be a nontrivial subgroup of the additive group of real numbers with the natural order. Let $V=V\left(\Gamma, R_{\gamma}\right)$ be the po-group constructed as in $\$ 2$. By Theorem 2.2, $V$ is an $l$-group. For each $\gamma$ in $\Gamma$ let

$$
V^{\gamma}=\left\{v \in V \mid v_{\alpha}=0 \text { for all } \alpha>\gamma\right\},
$$

and

$$
V_{\gamma}=\left\{v \in V \mid v_{\alpha}=0 \text { for all } \alpha \geqq \gamma\right\},
$$

THEOREM 6.1. The set

$$
\Delta=\left\{\left(V^{\gamma}, V_{\gamma}\right) \mid \gamma \in \Gamma\right\}
$$

is the minimal plenary subset of the set $\Gamma_{1}=\Gamma_{1}(V)$, and each element in $\Delta$ is special.

Proof. Clearly, $V^{\gamma}$ and $V_{\gamma}$ are $l$-ideals for all $\gamma$ in $\Gamma$. Consider $\delta \in \Gamma$, and let $v$ be an element in $V$ with $\delta$ th component nonzero and all other components zero. Then $v \in V^{\delta} \backslash V_{\delta}$, and if $C$ is an $l$-ideal of $V$ that does not contain $v$ and if $0<c \in C$, then $c_{\alpha}=0$ for all $\alpha \geqq \delta$, since otherwise $0<v \leqq n c$ for some integer $n>0$, and hence $v \in C$. Therefore $C \subseteq V_{\delta}$, and it follows that $V_{\delta}$ is the unique maximal $l$-ideal in $V$ without $v$. It is clear that $V^{\delta}$ covers $V_{\delta}$. Thus $\Delta$ is a subset of $\Gamma_{1}$ that consists of special elements.

To complete the proof, it suffices to show that $\Delta$ is a plenary set. If $0 \neq v \in V$, then $v \in V^{\gamma} \mid V_{\gamma}$ for each $\gamma$ such that $v_{\gamma}$ is a maximal component for $v$. If $v \notin V^{\alpha}$, then $v$ must have a maximal component $v_{\beta}$ with $\beta>\alpha$, and hence $v \in V^{\beta} \mid V_{\beta}$. Therefore $\Delta$ is plenary.

THEOREM 6.2. V has $a$ basis if and only if for each $\alpha \in \Gamma$ there exists $a \beta \in \Gamma$ such that $\alpha \geqq \beta$ and the elements in $\Gamma$ below $\beta$ form a chain.

This is an immediate consequence of Theorems 5.6 and 6.1.

Let $G$ be an abelian l-group, and let $\Delta$ be a plenary subset of $\Gamma_{1}=\Gamma_{1}(G)$. Let $P$ 
be a collection of maximal chains of $\Delta$, and let $\phi$ be a $v$-isomorphism of $G$ into $V\left(\Delta, G^{\delta} / G_{\delta}\right)$. As before, for each $M \in P$ let $V_{M}$ be the Hahn $o$-group

$$
V_{M}=\left\{v \in V \mid v_{\gamma}=G_{\gamma} \text { for all } \gamma \in \Delta \mid M\right\} .
$$

Let $L$ be the large cardinal sum of the $V_{M}$

and for each $M \in P$ let

$$
L=L\left(V_{M}: M \in P\right),
$$

$$
L_{M}=L\left(V_{Q}: M \neq Q \in P\right) .
$$

Let $\phi_{M}$ be the natural homorphism of $L$ into $L_{M}$.

If $\sigma$ is an $l$-isomorphism of an $l$-group $H$ onto a subdirect sum of $L$, then $H \sigma$ is said to be an irreducible representation of $H$ provided that $\sigma \phi_{M}$ is not one-to-one for all $M \in P$. For each $M \in P$ let $\pi_{M}$ be the projection of $V$ onto $V_{M}$ and for each $v \in V$ define

$$
v \pi=\left(\cdots, v \pi_{M}, \cdots\right) \in L
$$

It follows that if $\bigcup\{M \in P\}=\Delta$, then $\phi \pi$ is an $l$-isomorphism of $G$ onto a subdirect sum of the $V_{M}$ (see the definition of $v \vee \theta$ in the proof of Theorem 2.2).

THEOREM 6.3. Let $G$ be an abelian l-group, let $\Delta$ be a minimal plenary subset of $\Gamma_{1}$ and let $P$ consist of those maximal chains $M$ in $\Delta$ such that there is a $\beta \in M$ with $\{\alpha \leqq \beta \mid \alpha \in \Delta\}$ totally ordered. Let $\phi$ and $\pi$ be as above. Then the following are equivalent.

(1) $G$ has a basis.

(2) $\bigcup\{M \in P\}=\Delta$.

(3) $G \phi \pi$ is an irreducible representation of $G$.

Proof. By Theorem 5.6, (1) implies (2). Suppose that $\bigcup\{M \in P\}=\Delta$. Then $\phi \pi$ is an $l$-isomorphism of $G$ onto a subdirect sum of the $V_{M}$. Let $M \in P$. By assumption there is a $\beta \in M$ such that $\{\alpha \leqq \beta \mid \alpha \in \Delta\}$ is a chain. Since $\Delta$ is minimal, there is a $0 \neq g \in G$ such that all of the values of $g$ in $\Delta$ lie below $\beta$ (Theorem 5.1); that is, $g \phi \pi \phi_{M}=0$, and it follows that $G \phi \pi$ is irreducible and hence (2) implies (3).

Finally suppose that $G \phi \pi$ is an irreducible representation of $G$, and consider $\alpha \in \Delta$. There is a $0 \neq g \in G$ such that the values of $g$ in $\Delta$ lie below $\alpha$. Since $\phi \pi$ is an isomorphism, $g \phi \pi_{M} \neq 0$ for some $M \in P$. Thus some value $\beta$ of $g$ in $\Delta$ belongs to $M$. Therefore $\alpha \in M$ and hence $\Delta$ has property (*) of Theorem 5.6. Thus $G$ has a basis and so (3) implies (1).

RemarK. An abelian $l$-group $G$ has a basis if and only if it satisfies Jaffard's condition $M[10$, p. 231], and Jaffard has shown [10, Theorem 4, p. 251] that $G$ satisfies condition $M$ if and only if $G$ has an irreducible representation as a sublattice and a subdirect sum of a cardinal sum of $o$-groups. Thus we have re-proved part of Jaffard's result in our theory.

The following theorem was noted by Nakayama [15] for vector lattices. 
THEOREM 6.4. An abelian l-group $G$ is l-isomorphic to a subdirect sum of a large cardinal sum of additive groups of real numbers (with their natural order) if and only if for each $0 \neq g \in G$ there is an l-ideal of $G$ which is covered by $G$ and does not contain $g$, or equivalently, if and only if the intersection of the maximal l-ideals of $G$ is zero.

Proof. Let $R$ be the group of real numbers and let $G$ be a sublattice and a subdirect sum of a large cardinal sum of subgroups $R_{\gamma}$ of $R(\gamma \in \Gamma)$. We consider $G$ as a subgroup of $V\left(\Gamma, R_{\gamma}\right)$ where $\Gamma$ is trivially ordered. Then the identity map on $G$ is a $v$-isomorphism of $G$ into $V$. By the remarks preceding Theorem 5.10 for each $\gamma \in \Gamma$

$$
G(\gamma)=\left\{g=\left(\cdots, g_{\alpha}, \cdots\right) \mid g_{\gamma}=0\right\}
$$

is an $l$-ideal of $G$ and $G / G(\gamma)$ is $o$-isomorphic to $R_{\gamma}$. Thus if $0 \neq g \in G$ and $g_{\gamma} \neq 0$, then $G(\gamma)$ is an $l$-ideal of $G$ without $g$ and $G$ covers $G(\gamma)$.

Conversely, suppose that for each $0 \neq g \in G$ there is an $l$-ideal $C$ of $G$ which is covered by $G$ and does not contain $g$. In particular $G / C$ is $o$-isomorphic to a subgroup of $R$. Let $S$ be the collection of all $l$-ideals of $G$ that are covered by $G$. Then the mapping of $g \in G$ upon

$$
g \phi=(\cdots, g+C, \cdots), \quad C \in S
$$

is clearly an isomorphism of $G$ onto a subdirect sum of the large cardinal sum $Q$ of the $G / C$. The set $\{(G, C) \mid C \in S\}$ is a plenary subset of $\Gamma_{1}=\Gamma_{1}(G)$, and $\phi$ is a $v$-isomorphism. Therefore $G \phi$ is a sublattice of $Q$.

RemarK. The $l$-group $K$ described in Example 4 is Archimedean, but it does not satisfy the hypothesis of the preceding theorem. Thus it cannot be embedded as a subdirect sum and a sublattice of a cardinal sum of subgroups of $R$.

7. Examples. In this section we give some examples which illustrate the scope as well as the limitations of our theory. In general the method of construction is to start with the real numbers $R$ and a root system $\Delta$, and then to consider a subgroup and a sublattice $G$ of $V(\Delta, R)$. The usefulness of the examples is sometimes dependent on the fact that the set $\Delta$ reappears as a plenary subset of $\Gamma_{1}(G)$. This will happen whenever $G$ has the following properties:

(i) For each $\delta$ in $\Delta$ there exists an element $g$ in $G$ with $\delta$ as one of its maximal components.

(ii) $G \cap V_{\delta}$ is an $l$-ideal of $G$ that is maximal without $g$.

(iii) $V_{\alpha} \cap G \subseteq V_{\beta} \cap G$ implies $\alpha \leqq \beta$.

The reader can verify that these conditions are satisfied by the examples.

It follows from Theorem 5.7 that, if $G$ is an Archimedean $l$-group without a basis, then $\Gamma_{1}(G)$ contains no minimal plenary subset. Thus the group of all continuous functions on the unit interval $[0,1]$, with the usual ordering, is an 
Archimedean $l$-group with no minimal plenary subset. A smaller l-group with this property is provided by the following:

EXAMPLE 1 . Let $\Delta$ be the trivially ordered set of positive integers, and let $G$ consist of all those functions $f$ in $V(\Delta, R)$ which satisfy

(a) $f(i)$ is an integer for each $i$ in $\Delta$;

(b) there exists a positive integer $n=n(f)$ such that $f(i)=f(i+n)$ for all $i$ in $\Delta$.

Then $G$ is the set of all periodic integral valued functions. It is easy to show that $G$ is an Archimedean $l$-group. Moreover, $G$ has no basis, in fact, $G$ contains no basic elements. For let $g$ be a positive element in $G$ and choose an $i \in \Delta$ such that $g(i)>0$. Then the functions

$$
\begin{aligned}
& s(j)= \begin{cases}g(j) & \text { if } j \text { is a multiple of } 2 i \\
0 & \text { otherwise }\end{cases} \\
& t(j)= \begin{cases}g(j) & \text { if } j \text { is a multiple of } 3 i \\
0 & \text { otherwise }\end{cases}
\end{aligned}
$$

are both positive, less than $g$, and noncomparable. It follows that $G$ has no basic element, and hence $\Gamma_{1}(G)$ has no minimal plenary subset.

We next give an example of an $l$-group $G$ without a basis such that $\Gamma_{1}(G)$ has a unique minimal plenary subset $\Delta$ and yet no element of $\Delta$ is special. Thus for this group our embedding is minimal but there is no minimal Lorenzen embedding.

EXAMPLE 2. Let $I$ be the unit interval $[0,1]$ and for each positive integer $n$, let

$$
\left.I^{n}=I \times I \times \cdots \times I \text { ( } n \text { factors }\right) .
$$

Let $\Delta$ be the join of the $I^{n}$ for $n=1,2, \cdots$ partially ordered as follows:

$$
\left(x_{1}, \cdots, x_{m}\right)<\left(y_{1}, \cdots, y_{n}\right)
$$

if $m>n$ and $x_{i}=y_{i}$ for $i=1,2, \cdots, n$. In this case $V(\Delta, R)$ is the collection of all mappings of $\Delta$ into $R$. For each $\delta=\left(a_{1}, \cdots, a_{n}\right) \in \Delta$, let

$$
I_{\delta}=\left\{\left(a_{1}, \cdots, a_{n}, x\right) \in I^{n+1} \mid x \in I\right\} .
$$

Let $I_{\delta}$ have the usual topology as a subspace of $I^{n+1}$. Finally, let $G$ be the subgroup of $V(\Delta, R)$ consisting of all functions which are continuous on $I$ and $I_{\delta}$ for all $\delta \in \Delta$.

Thus every nonzero element of $G$ has infinitely many values, so that no element of $\Delta$ is special. However, $\Delta$ is a minimal plenary subset of $\Gamma_{1}(G)$ since for each $\delta \in \Delta$ there is an element of $G$, all of whose values lie below $\delta$ (see Theorem 5.1).

EXAMPLE 3. A divisible Archimedean $l$-group $G$ for which $V\left(\Gamma_{1}(G), G^{\gamma} / G_{\gamma}\right)$ is not Archimedean. Let $G$ be the $l$-group of all continuous functions on the half line $[0, \infty)$. Let

$$
J_{0}^{\prime}=\{f \in G \mid f \text { is bounded }\}
$$


Then $J_{0}^{\prime}$ is an $l$-ideal of $G$ and $f_{1}(x)=x$ does not belong to $J_{0}^{\prime}$. Let $J_{0}$ be an $l$-ideal of $G$ that contains $J_{0}^{\prime}$ and is maximal without $f_{1}$. By a simple inductive argument, $G$ contains an infinite ascending chain

$$
J_{0} \subset J_{1} \subset J_{2} \subset \cdots
$$

of $l$-ideals such that $J_{i}$ contains $f_{i}(x)=x^{i}$ and is maximal without $f_{i+1}(x)=x^{i+1}$. Thus, $\Gamma_{1}(G)$ contains an infinite ascending chain, and so, in particular, $V\left(\Gamma_{1}(G), G^{\gamma} / G_{\gamma}\right)$ is not Archimedean. In this case it is clearly possible to chose a plenary subset $\Delta$ of $\Gamma_{1}(G)$ such that $V\left(\Delta, G^{\delta} / G_{\delta}\right)$ is Archimedean. Namely, choose $\Delta=[0, \infty)$, the trivially-ordered root system we started with. The following example shows that this is not always possible.

EXAMPLE 4 (KAPLANSKY). Let $G$ consist of all real functions on $[0,1]$ of the form

$$
g(x)+\sum_{i=1}^{n} \frac{b_{i}}{\left(x-a_{i}\right)^{2}}
$$

where $g(x)$ is continuous. Define $f \in G$ to be positive if $f(x) \geqq 0$ for all $x \in[0,1]$ for which $f(x)$ is defined. Then $G$ is an Archimedean l-group and each maximal $l$-ideal of $G$ must contain all the continuous functions. In particular, an $l$-ideal of $G$ that does not contain $f(x)=1$ cannot be covered by $G$. Thus by Theorem $6.4, G$ is not a subdirect sum and a sublattice of a cardinal sum of subgroups of the reals.

It also follows that no plenary subset $\Delta$ of $\Gamma_{1}(G)$ can be trivially ordered. Thus $V\left(\Delta, G^{\delta} / G_{\delta}\right)$ is not Archimedean for any plenary subset of $\Gamma_{1}(G)$.

EXAMPLE 5. Let $\Delta$ be the set of all finite sequences of 1's and 2's, each of which starts with a 1. Partially order as in the following diagram:

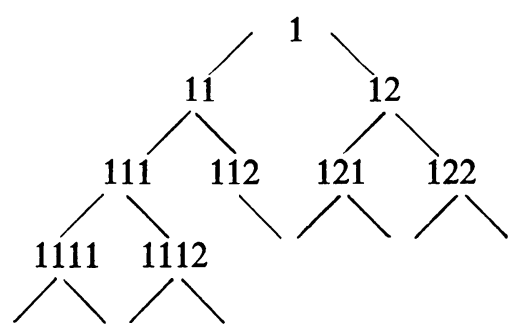

Then $G=V(\Delta, R)$ has no basis, but every element of $\Delta$ is special. Thus $\Delta$ is a minimal plenary subset of $\Gamma_{1}(G)$.

EXAMPLE 6 . For each positive integer $n$, let $R_{n}$ be the additive group of rationals and let $G$ be the small direct sum of the $R_{n}$,

$$
G=R_{1} \oplus R_{2} \oplus \cdots
$$

Lexicographically order $G$ from the left. Consider the mapping $\tau$ defined by 


$$
\begin{aligned}
(1,0,0, \cdots) & \rightarrow(1,1,1, \cdots) \\
(0,1,0,0, \cdots) & \rightarrow(0,1,0,1, \cdots) \\
(0,0,1,0,0, \cdots) & \rightarrow(0,0,1,0,0,1, \cdots) .
\end{aligned}
$$

The elements on the right are independent in the large direct sum $L$ of the $R_{n}$. Thus $\tau$ is induced by an isomorphism $\pi$ of $G$ into $L$. Clearly $(1,0,0, \cdots) \notin G \pi$, and hence $G \pi \neq G$.

Thus we have an $o$-group $G$ and $v$-isomorphisms $\pi$ and $\theta$ of $G$ into $V\left(\Gamma_{1}(G), G^{y} / G_{y}\right)$ (the large direct sum of the $R_{n}$ ) such that $G \theta$ contains the small direct sum of the $G^{\gamma} / G_{\gamma}$, but $G \pi$ does not.

8. Appendix. Let $G$ be an abelian divisible po-group and let $\Delta$ be a plenary subset of the set $\Gamma$ of all pairs $\left(G^{\gamma}, G_{y}\right)$ of convex subgroups of $G$ such that $G^{\gamma}$ covers $G_{\gamma}$. We have shown in Theorem 3.1 that there exists a $v$-isomorphism of $G$ into $V=V\left(\Delta, G^{\delta} / G_{\delta}\right)$. Here we derive a necessary and sufficient condition for the existence of a $v$-isomorphism $\phi$ of $G$ into $V$ such that $G \phi$ contains the small direct sum of the $G^{\delta} / G_{\delta}$. Even if such a $v$-isomorphism exists, it does not follow that all $v$-isomorphisms of $G$ into $V$ will have this property (see Example 6). Thus we must sharpen our proof of Theorem 3.1.

A set $T$ of subspaces $T_{\delta}(\delta \in \Delta)$ of $G$ is called a decomposition of $G$ provided that it has the following properties:

(1) $T_{\delta}+G^{\delta}=G$ for all $\delta \in \Delta$;

(2) $T_{\delta} \cap G^{\delta}=G_{\delta}$ for all $\delta \in \Delta$;

(3) for each $g \in G$, the set $\left\{\delta \in \Delta \mid g \notin T_{\delta}\right\}$ satisfies the maximum condition.

If $T$ is a decomposition of $G$, then the mapping of $g \in G$ upon

$$
g \widetilde{T}=\left(\cdots,\left(T_{\delta}+g\right) \cap G^{\delta}, \cdots\right)
$$

is a $v$-isomorphism of $G$ into $V$ [5, Theorem 2.4]. Conversely, if $\phi$ is a $v$-isomorphism of $G$ into $V$, then $\phi=\bar{T}$ for some decomposition $T$ of $G[5$, p. 12].

Let $T$ be a decomposition of $G$. Note that, if $\delta \in \Delta$ is a value of $g \in G$, then $g \notin T_{\delta}$, and $h \in T_{\delta}$ if and only if $(h \bar{T})_{\delta}=G_{\delta}$. For each $\alpha \in \Delta$ let $T_{\alpha}^{*}$ be the intersection of all the $T_{\delta}$ with the exception of $T_{\alpha}$. It can be shown that $G \bar{T}$ contains the small direct sum of the $G^{\delta} / G_{\delta}$ if and only if $T_{\delta}+T_{\delta}^{*}=G$ for all $\delta \in \Delta$, but this result is not very useful.

THEOREM 8.1. There exists a v-isomorphism $\phi$ of $G$ into $V$ such that $G \phi$ contains the small direct sum of the $G^{\delta} / G_{\delta}$ if and only if for each $\delta \in \Delta$

(*) each nonzero coset of $G^{\delta} / G_{\delta}$ contains an element whose only value in $\Delta$ is $\delta$.

Proof. Let $\phi$ be a $v$-isomorphism of $G$ into $V$ such that $G \phi$ contains the small direct sum of the $G^{\delta} / G_{\delta}$, and consider $\delta \in \Delta$ and $G_{\delta} \neq a+G_{\delta} \in G^{\delta} / G_{\delta}$. There exists 
$b \in G$ such that $(b \phi)_{\delta}=a+G_{\delta}$ and $(b \phi)_{\alpha}=G_{\alpha}$ for all $\alpha \neq \delta$. It is clear that $b$ is special with respect to $\Delta$.

Conversely suppose that $\Delta$ satisfies (*). We first show that for each $\delta \in \Delta$, $G^{\delta}=D_{\delta} \oplus G_{\delta}$ where $D_{\delta}$ is a group such that each nonzero element has the unique value $\delta$ in $\Delta$. For, if $B_{\delta}$ is a divisible group of elements such that each nonzero element has the unique value $\delta$ in $\Delta$ (in particular, $B_{\delta}=0$ will do), then $G_{\delta} \oplus B_{\delta} \subseteq G^{\delta}$. If $G^{\delta} \neq G_{\delta} \oplus B_{\delta}$, then there exists $g \in G^{\delta} \backslash G_{\delta} \oplus B_{\delta}$ with unique value $\delta$ in $\Delta$, and it follows by a straightforward argument that $G_{\delta} \oplus B_{\delta} \oplus R_{g} \subseteq G^{\delta}$ and each nonzero element in $B_{\delta} \oplus R_{g}$ has the unique value $\delta$ in $\Delta$, where $R_{g}$ is the one-dimensional subspace of $G$ that contains $g$.

Next, let $H$ be the small direct sum of the $D_{\delta}$, and for each $\delta \in \Delta$ let

$$
S_{\delta}=\sum_{\delta \neq \alpha \in \Delta} \oplus D_{\alpha}
$$

Then it is easy to check that the $S_{\delta}$ form a decomposition of $H$. By the existence theorem [5, p. 9] there is a decomposition $T$ of $G$ such that $S_{\delta} \subseteq T_{\delta}$ for all $\delta \in \Delta$. If $0 \neq d \in D_{\delta}$, then $d \in S_{\delta}^{*} \subseteq T_{\delta}^{*}$, and hence $(d \bar{T})_{\delta}=d+G_{\delta}$ and $(d \bar{T})_{\alpha}=G_{\alpha}$ for all $\delta \neq \alpha \in \Delta$. Therefore $G \bar{\Gamma}$ contains the small direct sum of the $G^{\delta} / G_{\delta}$. This completes the proof of the theorem.

Note that, if $\Delta$ is linearly ordered, then each element in $G$ has exactly one value in $\delta$, and hence (*) is satisfied. So we have the following corollary:

COROLlaRY 8.1. If $G$ is a divisible abelian o-group, then there exists a $v$-isomorphism $\phi$ of $G$ into the Hahn group $V\left(\Gamma, G^{\gamma} / G_{\gamma}\right)$ such that $G \phi$ contains the small direct sum of the $G^{\gamma} / G_{\gamma}$.

Finally, let $\pi$ be a Banaschewski function defined on the set of all subspaces of $G$, and let $\phi$ be the existent $v$-isomorphism of $G$ into $V=V\left(\Delta, G^{\delta} / G_{\delta}\right)$ (see the proof of Theorem 3.1). For each $\delta \in \Delta$ let

$$
T_{\delta}=G_{\delta}+\pi\left(G^{\delta}\right)
$$

Then it is easy to verify that the set $T$ of all the $T_{\delta}$ is a decomposition of $G$ and that $\phi=\bar{T}$, but it can be shown that not every $v$-isomorphism of $G$ into $V$ is induced by a Banaschewski function.

\section{REFERENCES}

1. B. Banaschewski, Totalgeordnete Moduln, Arch. Math. 7 (1956), 430-440.

2. G. Birkhoff, Lattice-ordered groups, Ann. of Math. (2) 43 (1942), 298-331.

3. — Lattice theory, rev. ed., Amer. Math. Soc. Colloq. Publ. Vol. 25, Amer. Math. Soc., Providence, R. I., 1948.

4. A. H. Clifford, Note on Hahn's theorem on ordered abelian groups, Proc. Amer. Math. Soc. 5 (1954), 860-683.

5. P. Conrad, Embedding theorems for abelian groups with valuations, Amer. J. Math. 75 (1953), 1-29. 
6. - - Some structure theorems for lattice-ordered groups, Trans. Amer. Math. Soc. 99 (1961), 212-240.

7. K. A. H. Gravett, Valued linear spaces, Quart. J. Math. Oxford Ser. (2)6(1955), 309-315.

8. —_, Metrics of sets and linear spaces, Quart. J. Math. Oxford Ser. (2) 10 (1959), 9-16.

9. H. Hahn, Ubber die nichtarchimedischen Grössensysteme, S.-B. Kaiserlichen Akad. Wiss. Math. Nat. Kl. IIa, 116 (1907), 601-655.

10. P. Jaffard, Contribution a l'étude des groupes ordonées, J. Math. Pure Appl. 32 (1953), 203-280.

11. - Extension des groupes réticulés et applications, Publ. Sci. Univ. Alger. Sér. A 1 (1954), 197-222.

12. - Sur le spectre d'un groupe réticulé et l-unicité des réalisations irreductibles, Ann. Univ. Lyon Sect. A 22 (1959), 43-47.

13. P. Lorenzen, Abstrakte Begründung der multiplikativen Idealtheorie, Math. Z. 45 (1939), 533-553.

14. — Ubber halbgeordnete Gruppen, Math. Z. 52 (1949), 484-526.

15. K. Nakayama, Note on lattice-ordered groups, Proc. Imp. Acad. Tokyo 18 (1942), 1-4.

16. P. Ribenboim, Conjonction d'ordres dans les groupes abeliens ordonées, An. Acad. Brasil. Ci. 29 (1957), 201-224.

17. _- Un théorème de réalisation de groupes réticulés, Pacific J. Math. 10 (1960), 305-308.

18. F. Sik, Über Summen einfach geordneter Gruppen, Czechoslovak Math. J. 8 (1953), 22-53.

19. - Uber subdirekte Summen geordneter Gruppen, Czechoslovak Math. J. 10(1960), $400-424$.

TULANE UNIVERSITY,

New Orleans, Louisiana 